Article

\title{
Optimization and Analysis of Multilayer Planar Spiral Coils for the Application of Magnetic Resonance Wireless Power Transfer to Wearable Devices
}

\author{
Young-Jin Park ${ }^{1, * \mathbb{D}}$, Ji-Eun Kim ${ }^{1}$, Kyung-Min $\mathrm{Na}^{2}$, Ki-Dong Yang ${ }^{1}$ and Kyung-Hwan Cho ${ }^{1}$ \\ 1 Korea Electrotechnology Research Institute, An-San 15588, Korea; jeeeungim@keri.re.kr (J.-E.K.); \\ kdyang@keri.re.kr (K.-D.Y.); chokh@keri.re.kr (K.-H.C.) \\ 2 Eta Electronics R\&D, Gangnam-gu, Seoul 06121, Korea; kyungmin.na@etaelec.com \\ * Correspondence: yjpark@keri.re.kr
}

Citation: Park, Y.-J.; Kim, J.-E.; Na, K.-M.; Yang, K.-D.; Cho, K.-H. Optimization and Analysis of Multilayer Planar Spiral Coils for the Application of Magnetic Resonance Wireless Power Transfer to Wearable Devices. Energies 2021, 14, 5113.

https://doi.org/10.3390/en14165113

Academic Editor: Nicu Bizon

Received: 20 July 2021

Accepted: 12 August 2021

Published: 19 August 2021

Publisher's Note: MDPI stays neutral with regard to jurisdictional claims in published maps and institutional affiliations.

Copyright: (c) 2021 by the authors. Licensee MDPI, Basel, Switzerland. This article is an open access article distributed under the terms and conditions of the Creative Commons Attribution (CC BY) license (https:// creativecommons.org/licenses/by/ $4.0 /)$.

\begin{abstract}
In this study, small multilayer planar spiral coils were analyzed and optimized to wirelessly charge an in-ear wearable bio-signal monitoring device in a wine-glass-shaped transmitter (Tx) based on magnetic resonance wireless power transfer (MR-WPT). For analysis of these coils, a volume filament model (VFM) was used, and an equivalent circuit formulation for the VFM was proposed. The proposed method was applied to design effective multilayer coils with a diameter and height of 6 and $3.8 \mathrm{~mm}$, respectively, in the wearable device. For the coils, a printed circuit board having a $0.6 \mathrm{~mm}$ thick dielectric substrate and a $2 \mathrm{oz}$ thick copper metal was used. Moreover, the coils on each layer were connected in series. The dimensions of the double-, four-, and eight-layer coils were optimized for the maximum quality factor ( $Q$-factor) and coupling efficiency. The operating frequency was $6.78 \mathrm{MHz}$. The optimal dimensions for the maximum Q-factor varied depending on the number of coil layers, pattern width, and turn number. For verification, the designed coils were fabricated and measured. For the four-layer coil, the coupling efficiency and Q-factor using the measured resistance and mutual inductance were $58.1 \%$ and 32.19 , respectively. Calculations showed that the maximum Q-factor for the four-layer coil was 40.8 and the maximum coupling efficiency was $60.1 \%$. The calculations and measurement were in good agreement. Finally, the entire system of the in-ear wearable bio-signal monitoring device, comprising a wine-glass-shaped transmitter, the designed receiving coil, and a monitoring circuit, was fabricated. The measured dc-dc efficiency of the MR-WPT system was $16.08 \%$.
\end{abstract}

Keywords: magnetic resonance wireless power transfer (MR-WPT); planar spiral coil; volume filament model (VFM); wearable devices; planar multilayer coil

\section{Introduction}

Recently, magnetic resonance wireless power transfer (MR-WPT) in the MHz frequency range has been applied to wirelessly charge wearable Internet-of-Things (IoT) devices, such as wearable electronic devices and advanced biomedical implant devices. Compact effective coils are essential for the application of wireless power transfer in these devices, and for cases wherein the Rx coil is much smaller than the Tx coil [1-3].

For wearable application, a planar spiral coil is ideal for use due to its compactness and easy fabrication. In [4], a simple fabrication method by printing with a metal ink dispenser was reported. In [5,6], the optimal design method for a printed spiral coil was reported. Moreover, for the application in small implantable systems, a double-layer planar spiral coil, made by interconnecting two identical eight turn planar spiral coils, was used at $6.78 \mathrm{MHz}$ [7]. The small planar Rx coil dimension was about $15 \mathrm{~mm}(\mathrm{~W}) \times 10 \mathrm{~mm}(\mathrm{~L})$ and the metal thickness was $0.035 \mathrm{~mm}$, whereas the dimensions of a helical three-turn Tx coil were $39.6 \mathrm{~cm}(\mathrm{~W}) \times 34.6 \mathrm{~cm}(\mathrm{~L}) \times 8 \mathrm{~cm}(\mathrm{H})$. The dc-dc efficiency of the system was less than $1 \%$. Moreover, as a potential application, the proof-of-concept of a fully wireless 
rechargeable implant system in a human brain was demonstrated. It is shown that it is difficult to make small wearable and implant WPT systems having good efficiency in the case that the Rx coil is much smaller than the Tx coil.

In [8], the AC loss of a circular planar coil was analyzed. The loss consisted of conduction loss, including the skin effect and the proximity loss. Furthermore, the pattern width and metal thickness were normalized by the skin depth. However, these methods are inapplicable for multilayer planar spiral coils.

In [9], a double-layer planar spiral coil was studied for MR-WPT. Both the partial element equivalent circuit method (PEEC) and finite element method (FEM) were used to determine the resonant frequency, and the coil losses were analyzed to obtain a higher quality factor (Q-factor). However, the design and optimization of the coil parameters for the maximum $\mathrm{Q}$-factor and maximum coupling efficiency were not provided under the conditions of a target frequency and the limitation of the maximum size.

In [10], a method deriving a resonant frequency and the Q-factor of a four-layer printed spiral coil, using coil dimensions such as turn number, line width, and number of layers, was provided. However, optimization of the coil dimensions for the maximum Q-factor and coupling efficiency was not provided with the limitation of the coil dimensions.

In [11], a 12-layer planar spiral coil, comprising a Tx coil and four flexible Rx coils of a single layer, were reported for wearable applications. The system showed less influence of efficiency according to the deformation and misalignment between the Tx and Rx coils. However, the optimization of the coil dimensions for the maximum Q-factor and coupling efficiency was not explained.

In the current paper, a small multilayer planar spiral coil was analyzed using the volume filament model (VFM) in the MHz frequency range to assess the applicability of the coil for wearable bio-signal monitoring devices. In particular, the design process and analysis results are reported for the case in which the Tx coil is larger than the Rx coil in wearable and implantable devices. The operating frequency was $6.78 \mathrm{MHz}$. By considering the size limitation of wearable devices, a multilayer coil was optimized for maximum coupling efficiency. Moreover, the coils were optimized for the maximum Q-factor.

This paper is organized as follows. In Section 2, a wireless power transfer system for a compact wearable bio-signal monitoring device is proposed. In Section 3, for the optimal design of the Rx coil, the VFM and equivalent circuit formulation of the double-layer planar spiral coil is presented. Section 4 outlines the fabrication of the designed coils, and measurement results are verified with calculations. Finally, the complete bio-signal monitoring device is integrated and tested.

\section{Proposed Wireless Power Transfer System for a Wearable Bio-Signal Monitoring Device}

In Figure 1, the proposed wireless power transfer system is displayed. As shown in Figure 1b, a Tx circuit with a high-frequency inverter and Bluetooth low energy (BLE) module for charging control is placed at the base. As shown in Figure 1c, as a Tx coil, a bowl-shaped helix copper wire coil is used, which is equivalent to a concentric multi-loop helix coil. Therefore, the wearable bio-signal monitoring Rx device can be charged by simply placing it in the bowl. In addition, an Rx coil with a multilayer planar spiral coil is integrated with the printed circuit board (PCB) board of the device.

Figure $2 \mathrm{a}$ illustrates the double-layer and Figure $2 \mathrm{~b}$ four-layer planar spiral coils that are used in the proposed system. The four-layer planar spiral coils are connected in series and consist of two PCBs. Each PCB has two spiral coils connected in series. The dielectric PCB substrate thickness is $0.6 \mathrm{~mm}$, and the metal thickness is $2 \mathrm{oz}$. For the integration of the coil with a wearable bio-signal monitoring device, the maximum coil size is limited to $7 \times 7 \times 3.8 \mathrm{~mm}$, with the distance between the two PCBs being $2.3 \mathrm{~mm}$. The coil radius, $R R$, is $3 \mathrm{~mm}$. 


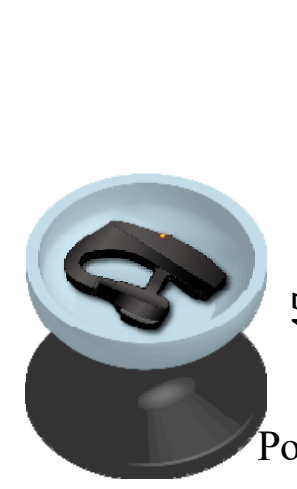

(a)

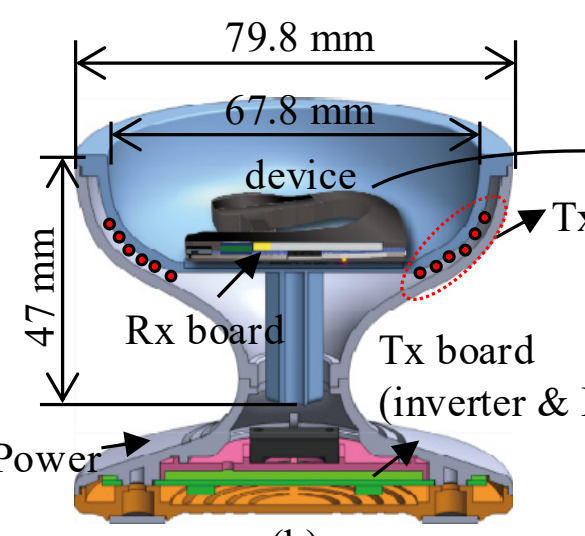

(b)

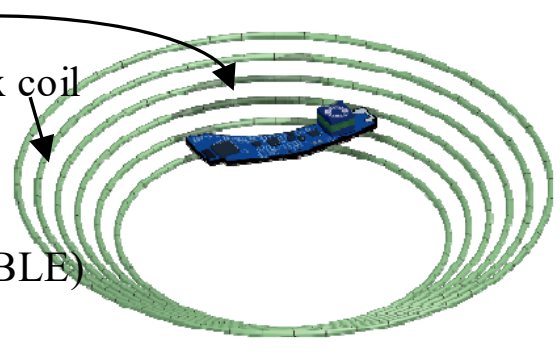

(c)

Figure 1. (a) Illustration of the wireless charging station of a wearable bio-signal monitoring device; (b) the cross-sectional view; (c) the transmitting coil used in the charging station.

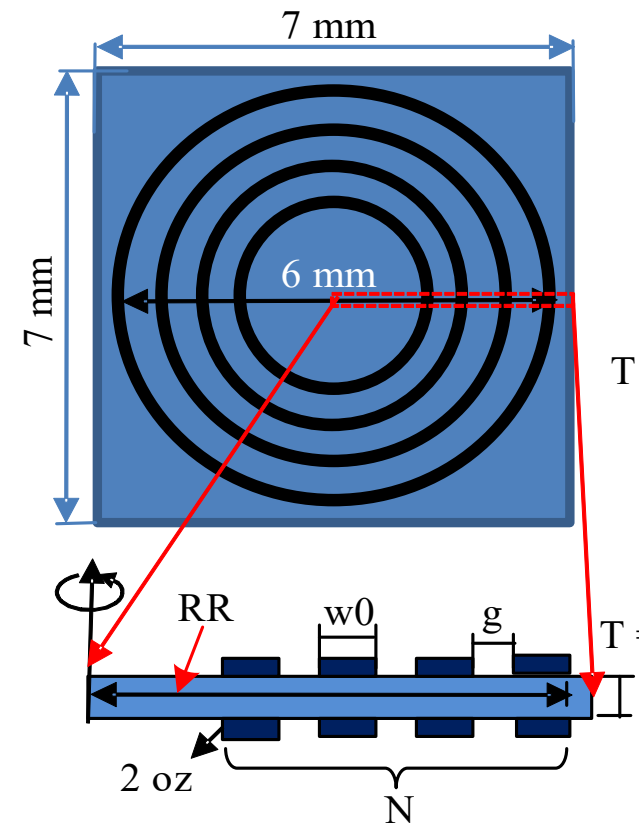

(a)

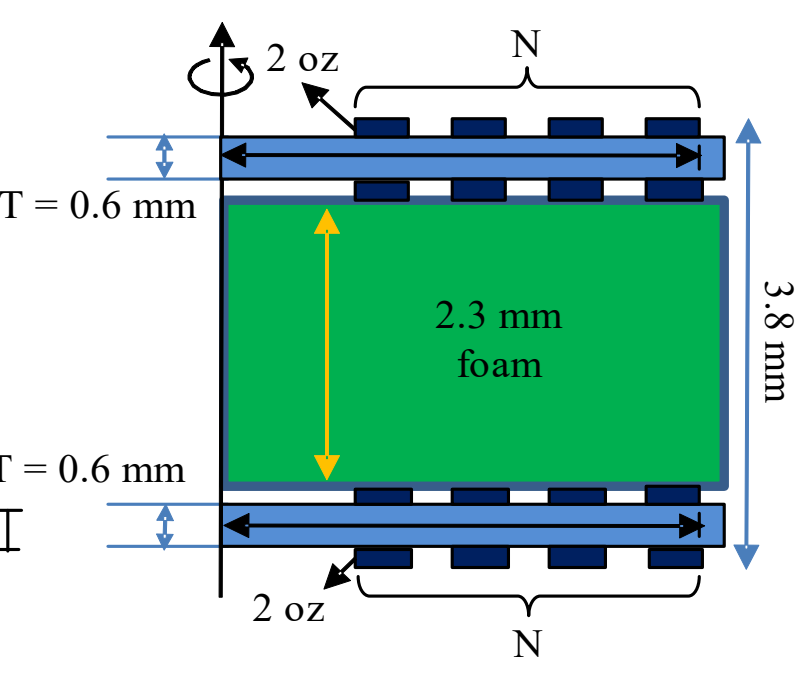

(b)

Figure 2. Schematic of a compact multilayer planar spiral coil, with the cross-sectional views of (a) the double-layer and (b) four-layer spiral coils.

\section{Calculation of Resistance and Inductance of the Multilayer Planar Spiral Coil}

3.1. VFM and Equivalent Circuit Formulation for a Multilayer Planar Spiral Coil

In Figure $3 \mathrm{a}$, the cross section of a $n$-loop planar coil is shown. It is noted that the $n$-loop double layer planar coil is equivalent to a double-layer planar spiral coil of $n$ turns for simple calculation. The PCB thickness is $T$, the pattern width is $w 0$, the gap is $g$, the metal thickness is $t$, and the turn number is $n$. The upper and bottom loops are aligned and connected in series, i.e., the $k^{\text {th }}$ loop has an upper loop, the $s k U^{\text {th }}$ loop, and a bottom loop, the $s k D^{\text {th }}$ loop. The net currents in each loop are identical.

For analysis, each loop is discretized into circular filament loops having a square cross section, with the square width, $d$, being less than $0.3 \delta$, where $\delta$ is the skin depth, as shown in Figure $3 \mathrm{~b}$. In Figure $3 \mathrm{~b}$, the $s k U^{\text {th }}$ and $s m D^{\text {th }}$ loops are chosen. The number of the total mesh of each loop is $n$. 


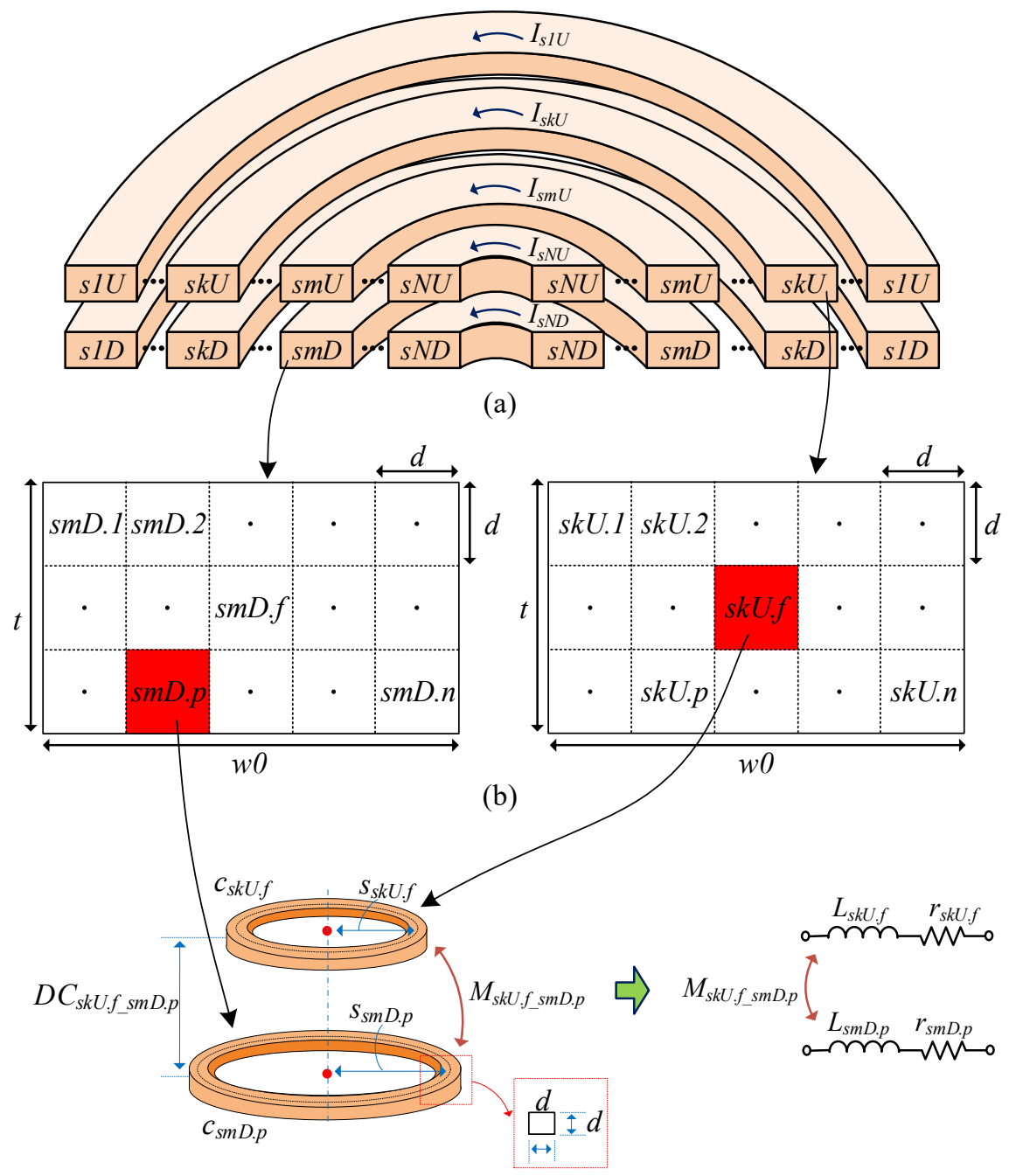

(c)

Figure 3. (a) Schematic of the double-layer multi-loop planar spiral coil; (b) the discretization for the volume filament model (VFM); (c) two filament loops and their equivalent circuit.

As shown in Figure 3c, two circular filament loops of the $f^{\text {th }}$ filament loop of the $s k U^{\text {th }}$ loop, $c_{s k U . f}$, and the $p^{\text {th }}$ filament loop of the $s m D^{\text {th }}$ loop, $c_{s m D . p}$, are chosen. $M_{s k U . f_{s} s m . p}$ is the mutual inductance between the two filament loops $c_{s k U}$.f and $c_{s m D . p}$. In addition, the two loops can be represented in terms of resistance $\left(\mathrm{r}_{s k U . f}, \mathrm{r}_{s m D . p}\right)$ and inductance $\left(L_{s k U . f}\right.$, $\left.L_{s m D . p}\right)$ of $c_{s k U . f}$ and $c_{s m D . p}$ and mutual inductance, as shown in Figure 3c.

In Figure 4, the equivalent circuit of the double-layer planar spiral coil of $n$ turns that was used for analysis using the VFM is displayed. Each loop is represented with resistance and self-inductance of $n$ filament loops, which are discretized in parallel. For example, the $s k U^{\text {th }}$ and $s m D^{\text {th }}$ loops in Figure 3 are represented with the equivalent circuits of $s k U^{t h}$ and $s m D^{\text {th }}$ loops, which are equivalent to the resistance and inductance of $n$ filament loops in Figure 4, respectively. Additionally, mutual inductances between two filament loops are included. For example, $M_{\text {skU.f_smu.p }}, M_{s k U . f_{-} s m D . p}, M_{s k D . f \_s m u . p}$, and $M_{s k D . f \_s m D . p}$ are the mutual inductances between two filament loops of $c_{s k U . f}$ and $c_{s m U . p}, c_{s k U . f}$ and $c_{s m D . p}, c_{s k D . f}$ and $c_{s m U . p}$, and $c_{s k D . f}$ and $c_{s m D . p}$, respectively. It should also be noted that the filament loop, $c_{s k U . f}$, is represented by resistance $r_{s k U . f}$ and inductance $L_{s k U . f}$. Mutual inductances between two loops are represented in matrix form. For example, $\boldsymbol{M}_{s k U \_s m U}, \boldsymbol{M}_{s k U \_s m D}, \boldsymbol{M}_{s k D \_s m U}$, and $\boldsymbol{M}_{s k D \_s m D}$ are the mutual inductance matrices between two loops of $s k U^{\text {th }}$ and $s m U^{\text {th }}$, $s k U^{t h}$ and $s m D^{t h}, s k D^{t h}$ and $s m U^{t h}$, and $s k D^{t h}$ and $s m D^{t h}$, respectively. 

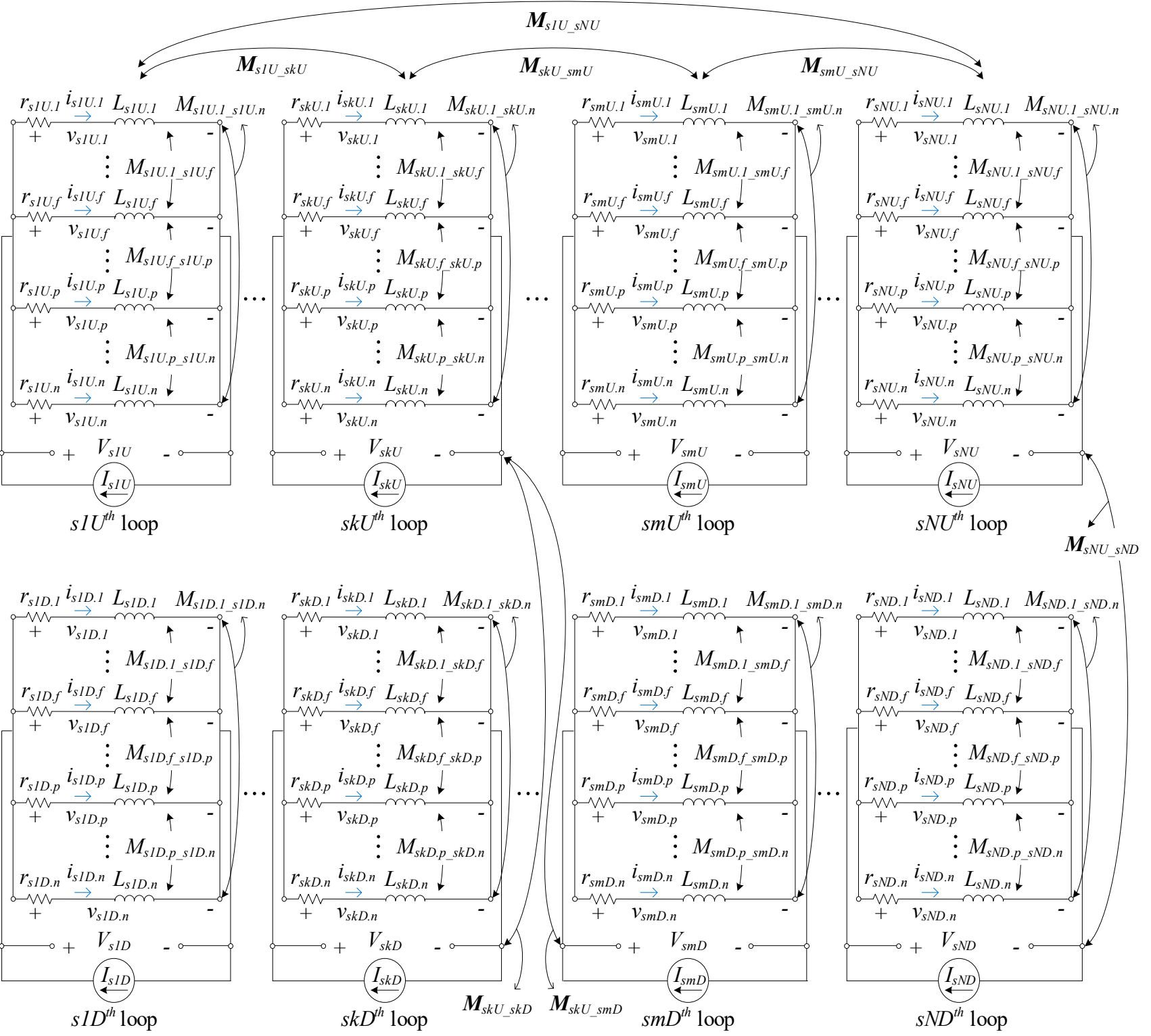

Figure 4. The equivalent circuit of the double-layer planar spiral coil of $n$ turns shown in Figure 3 for analysis using the VFM.

The net current of the $s k U^{t h}$ loop is $I_{s k U}$, and $i_{s k U . f}$ is the current of the filament loop, $c_{s k U . f}$.

From the equivalent circuit, $V_{s k U}$ is the voltage of the $s k U^{\text {th }}$ loop and $v_{s k U . f}$ is the voltage of the filament loop, $c_{s k U . f}$. Because each loop is connected in series,

$$
V_{s k U}=v_{s k U .1}=\cdots=v_{s k U . f}=\cdots=v_{s k U . p}=\cdots=v_{s k U . n} .
$$

By applying Kirchhoff's voltage law to the circuit,

$$
V_{T}=X I_{T}
$$

where

$$
\begin{gathered}
\boldsymbol{V}_{T}=\left[\begin{array}{lllllll}
\boldsymbol{V}_{s 1} & \cdots & \boldsymbol{V}_{s k} & \cdots & \boldsymbol{V}_{s m} & \cdots & \boldsymbol{V}_{s N}
\end{array}\right]^{T}, \\
\boldsymbol{I}_{T}=\left[\begin{array}{lllllll}
\boldsymbol{I}_{s 1} & \cdots & \boldsymbol{I}_{s k} & \cdots & \boldsymbol{I}_{s m} & \cdots & \boldsymbol{I}_{s N}
\end{array}\right]^{T}, \\
\boldsymbol{V}_{s k}=\boldsymbol{V}_{s k U}+\boldsymbol{V}_{s k D}, \boldsymbol{I}_{s k}=\boldsymbol{I}_{s k U}+\boldsymbol{I}_{s k D},
\end{gathered}
$$




$$
\begin{aligned}
& \boldsymbol{V}_{s k U}=\left[\begin{array}{lllll}
v_{s k U .1} & \cdots & v_{s k U . f} & \cdots & v_{s k U . n}
\end{array}\right], \boldsymbol{V}_{s k D}=\left[\begin{array}{lllll}
v_{s k D .1} & \cdots & v_{s k D . f} & \cdots & v_{s k D . n}
\end{array}\right], \\
& \boldsymbol{I}_{s k U}=\left[\begin{array}{lllll}
i_{s k U .1} & \cdots & i_{s k U . f} & \cdots & i_{s k U . n}
\end{array}\right]=\boldsymbol{I}_{s k D}=\left[\begin{array}{lllll}
i_{s k D .1} & \cdots & i_{s k D . f} & \cdots & i_{s k D . n}
\end{array}\right], \\
& \boldsymbol{X}=\left[\begin{array}{ccccccc}
\boldsymbol{R}_{s 1}+j \omega \boldsymbol{M}_{s 1 \_s 1} & \cdots & j \omega \boldsymbol{M}_{s 1 \_s k} & \cdots & j \omega \boldsymbol{M}_{s 1 \_s m} & \cdots & j \omega \boldsymbol{M}_{s 1 \_s N} \\
\vdots & \ddots & \vdots & \ddots & \vdots & \ddots & \vdots \\
j \omega \boldsymbol{M}^{T}{ }_{s 1 \_s k} & \cdots & \boldsymbol{R}_{s k}+j \omega \boldsymbol{M}_{s k \_s k} & \cdots & j \omega \boldsymbol{M}_{s k \_s m} & \cdots & j \omega \boldsymbol{M}_{s k \_s N} \\
\vdots & \ddots & \vdots & \ddots & \vdots & \ddots & \vdots \\
j \omega \boldsymbol{M}^{T}{ }_{s 1 \_s m} & \cdots & j \omega \boldsymbol{M}^{T}{ }_{s k \_s m} & \cdots & \boldsymbol{R}_{s m}+j \omega \boldsymbol{M}_{s m \_s m} & \cdots & j \omega \boldsymbol{M}_{s m_{\_} s N} \\
\vdots & \ddots & \vdots & \ddots & \vdots & \ddots & \vdots \\
j \omega \boldsymbol{M}^{T}{ }_{s 1 \_s N} & \cdots & j \omega \boldsymbol{M}^{T}{ }_{s k \_s N} & \cdots & j \omega \boldsymbol{M}^{T}{ }_{s m \_s N} & \cdots & \boldsymbol{R}_{s N}+j \omega \boldsymbol{M}_{s N \_s N}
\end{array}\right]
\end{aligned}
$$

Here, $\omega$ is the angular frequency and $[\cdot]^{T}$ is the transverse matrix of $[\cdot]$. The impedance matrix, $X$, consists of the resistance matrix and the mutual inductance matrix. The resistance matrix $\boldsymbol{R}_{s k}$ and the mutual inductance matrix $\boldsymbol{M}_{s k_{-} s m}$ are defined as follows:

$$
\begin{gathered}
\boldsymbol{R}_{s k}=\left[\begin{array}{cc}
\boldsymbol{R}_{s k U} & 0 \\
0 & \boldsymbol{R}_{s k D}
\end{array}\right], \\
\boldsymbol{M}_{s k_{\_} s m}=\left[\begin{array}{ll}
\boldsymbol{M}_{s k U \_s m U} & \boldsymbol{M}_{s k U_{\_} s m D} \\
\boldsymbol{M}_{s k D \_s m U} & \boldsymbol{M}_{s k D \_s m D}
\end{array}\right],
\end{gathered}
$$

where

$$
\begin{aligned}
\boldsymbol{R}_{s k U} & =\left[\begin{array}{ccccccc}
r_{s k U .1} & \cdots & 0 & \cdots & 0 & \cdots & 0 \\
\vdots & \ddots & \vdots & \ddots & \vdots & \ddots & \vdots \\
0 & \cdots & r_{s k U . f} & \cdots & 0 & \cdots & 0 \\
\vdots & \ddots & \vdots & \ddots & \vdots & \ddots & \vdots \\
0 & \cdots & 0 & \cdots & r_{s k U . p} & \cdots & 0 \\
\vdots & \ddots & \vdots & \ddots & \vdots & \ddots & \vdots \\
0 & \cdots & 0 & \cdots & 0 & \cdots & r_{s k U . n}
\end{array}\right], \\
\boldsymbol{R}_{s k D} & =\left[\begin{array}{ccccccc}
r_{s k D .1} & \cdots & 0 & \cdots & 0 & \cdots & 0 \\
\vdots & \ddots & \vdots & \ddots & \vdots & \ddots & \vdots \\
0 & \cdots & r_{s k D . f} & \cdots & 0 & \cdots & 0 \\
\vdots & \ddots & \vdots & \ddots & \vdots & \ddots & \vdots \\
0 & \cdots & 0 & \cdots & r_{s k D . p} & \cdots & 0 \\
\vdots & \ddots & \vdots & \ddots & \vdots & \ddots & \vdots \\
0 & \cdots & 0 & \cdots & 0 & \cdots & r_{s k D . n}
\end{array}\right],
\end{aligned}
$$

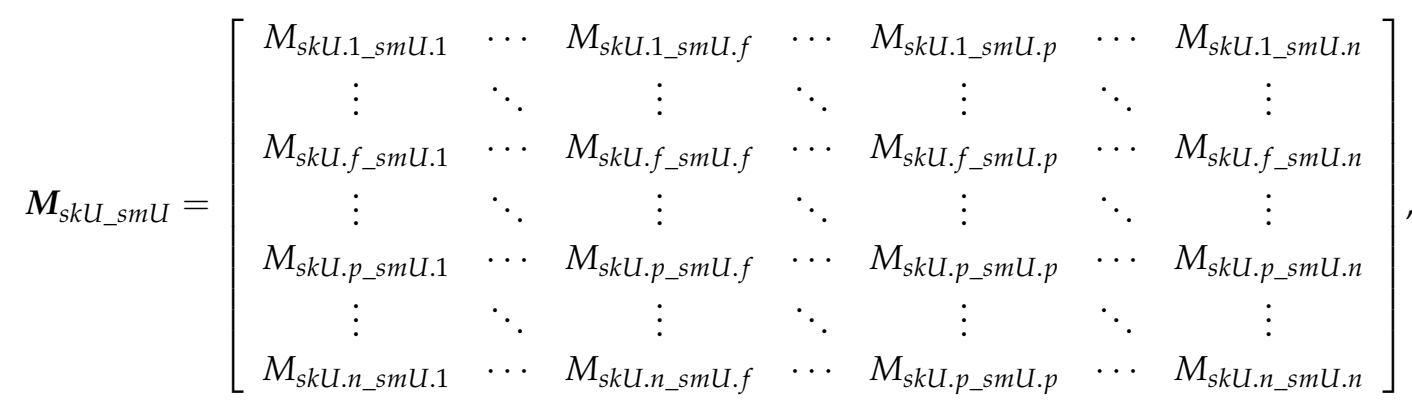




$$
\begin{aligned}
& M_{s k U \_s m D}=\left[\begin{array}{ccccccc}
M_{s k U .1 \_s m D .1} & \cdots & M_{s k U .1 \_s m D . f} & \cdots & M_{s k U .1 \_s m D . p} & \cdots & M_{s k U .1 \_s m D . n} \\
\vdots & \ddots & \vdots & \ddots & \vdots & \ddots & \vdots \\
M_{s k U . f \_s m D .1} & \cdots & M_{s k U . f \_s m D . f} & \cdots & M_{s k U . f \_s m D . p} & \cdots & M_{s k U . f \_s m D . n} \\
\vdots & \ddots & \vdots & \ddots & \vdots & \ddots & \vdots \\
M_{s k U . p \_s m D .1} & \cdots & M_{s k U . p \_s m D . f} & \cdots & M_{s k U . p \_s m D . p} & \cdots & M_{s k U . p \_s m D . n} \\
\vdots & \ddots & \vdots & \ddots & \vdots & \ddots & \vdots \\
M_{s k U . n \_s m D .1} & \cdots & M_{s k U . n \_s m D . f} & \cdots & M_{s k U . p \_s m D . p} & \cdots & M_{s k U . n \_s m D . n}
\end{array}\right] \text {, } \\
& M_{s k D \_s m U}=\left[\begin{array}{ccccccc}
M_{s k D .1 \_s m U .1} & \cdots & M_{s k D .1 \_s m U . f} & \cdots & M_{s k D .1 \_s m U . p} & \cdots & M_{s k D .1 \_s m U . n} \\
\vdots & \ddots & \vdots & \ddots & \vdots & \ddots & \vdots \\
M_{s k D . f \_s m U .1} & \cdots & M_{s k D . f \_s m U . f} & \cdots & M_{s k D . f \_s m U . p} & \cdots & M_{s k D . f \_s m U . n} \\
\vdots & \ddots & \vdots & \ddots & \vdots & \ddots & \vdots \\
M_{s k D . p \_s m U .1} & \cdots & M_{s k D . p \_s m U . f} & \cdots & M_{s k D . p \_s m U . p} & \cdots & M_{s k D . p \_s m U . n} \\
\vdots & \ddots & \vdots & \ddots & \vdots & \ddots & \vdots \\
M_{s k D . n \_s m U .1} & \cdots & M_{s k D . n \_s m U . f} & \cdots & M_{s k D . p \_s m U . p} & \cdots & M_{s k D . n \_s m U . n}
\end{array}\right] \text {, } \\
& M_{s k D \_s m D}=\left[\begin{array}{ccccccc}
M_{s k D .1 \_s m D .1} & \cdots & M_{s k D .1 \_s m D . f} & \cdots & M_{s k D .1 \_s m D . p} & \cdots & M_{s k D .1 \_s m D . n} \\
\vdots & \ddots & \vdots & \ddots & \vdots & \ddots & \vdots \\
M_{s k D . f \_s m D .1} & \cdots & M_{s k D . f \_s m D . f} & \cdots & M_{s k D . f \_s m D . p} & \cdots & M_{s k D . f \_s m D . n} \\
\vdots & \ddots & \vdots & \ddots & \vdots & \ddots & \vdots \\
M_{s k D . p \_s m D .1} & \cdots & M_{s k D . p \_s m D . f} & \cdots & M_{s k D . p \_s m D . p} & \cdots & M_{s k D . p \_s m D . n} \\
\vdots & \ddots & \vdots & \ddots & \vdots & \ddots & \vdots \\
M_{s k D . n \_s m D .1} & \cdots & M_{s k D . n \_s m D . f} & \cdots & M_{s k D . p \_s m D . p} & \cdots & M_{s k D . n \_s m D . n}
\end{array}\right] \text {, }
\end{aligned}
$$

$\boldsymbol{R}_{s k U}$ and $\boldsymbol{R}_{s k D}$ are the diagonal matrices of the resistance of $n$ filament loops of the $s k U^{t h}$ and $s k D^{\text {th }}$ loops, as shown in Figure 4, respectively. $\boldsymbol{M}_{s k U \_s m U}, \boldsymbol{M}_{s k U \_s m D}, \boldsymbol{M}_{s k D \_s m U}$, and $\boldsymbol{M}_{s k D \_s m D}$ are the matrixes of $M_{s k U . f_{-} s m U . p}, M_{s k U . f_{-} s m D . p}, M_{s k D . f_{-} s m U . p}$, and $M_{s k D . f_{-} s m D . p}$ with $k, m=1, \cdots, n$ and $f, p=1, \cdots, n$, respectively. When $k=m$ and $f=p, M_{\text {skU.f.smu.p }}$ and $M_{s k D . f_{-} s m D . p}$ become the self-inductances of $c_{s k U . f}, L_{s k U . f}$, and $c_{s k D . f}, L_{s k D . f}$, respectively.

The current distribution of each filament loop can be assumed to be uniform [12]; thus, the resistance of $c_{s k U . f}, r_{s k U . f}$, can be written as follows:

$$
r_{s k U . f}=\frac{2 \pi s_{s k U . f}}{\sigma d^{2}}
$$

where $\sigma$ is the conductivity of copper.

The inductance of $c_{s k U . f}, L_{s k U . f}$, is calculated as follows:

$$
L_{s k U . f}=\mu_{0} s_{s k U . f}\left[\ln \frac{8 s_{s k U . f}}{(d / 2)}-1.75\right] .
$$

Here, $\mu_{0}$ is the permeability in vacuum.

The mutual inductance, $M_{s k U . f \_s m D . p}$, is given by the following equation:

$$
M_{\text {skU.f_smD.p }}=\mu_{0} \sqrt{s_{\text {skU.f. }} S_{\text {smD.p }}}\left[\left(\frac{2}{\kappa}-2\right) K(\kappa)-\frac{2}{\kappa} E(\kappa)\right],
$$

where

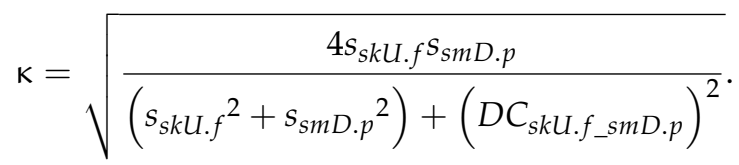


$K(\kappa)$ and $E(\kappa)$ are the complete elliptic integrals of the first and second kind, respectively [13].

From the equivalent circuit, the net currents of the loops are identical and can be expressed as follows:

$$
I_{s 1 U}=I_{s 1 D}=\cdots=I_{s k U}=I_{s k D}=\cdots=I_{s N U}=I_{s N D},
$$

where

$$
I_{s k U}=\sum_{f=1}^{n} i_{s k U . f}, \quad I_{s k D}=\sum_{f=1}^{n} i_{s k D . f} .
$$

Therefore, $\boldsymbol{V}_{T}$ and $\boldsymbol{I}_{T}$ can be written as follows:

$$
\begin{gathered}
\boldsymbol{V}_{T}=\boldsymbol{F}_{s 1 U}{ }^{T} V_{s 1 U}+\boldsymbol{F}_{s 1 D}{ }^{T} V_{s 1 D}+\cdots+\boldsymbol{F}_{s k U}{ }^{T} V_{s k U}+\cdots+\boldsymbol{F}_{s N D}{ }^{T} V_{s N D}, \\
\boldsymbol{I}_{T}=\boldsymbol{F}_{s 1 U}{ }^{T} I_{s 1 U}+\boldsymbol{F}_{s 1 D}{ }^{T} I_{s 1 D}+\cdots+\boldsymbol{F}_{s k U}{ }^{T} I_{s k U}+\cdots+\boldsymbol{F}_{s N D}{ }^{T} I_{s N D},
\end{gathered}
$$

where

$$
\begin{aligned}
& \boldsymbol{F}_{s k U, D}=\left[\begin{array}{llllll}
\mathbf{1}_{n}{ }^{T} \delta_{s 1 U_{-} s k U} & \mathbf{1}_{n}{ }^{T} \delta_{s 1 D_{-} s k D} & \cdots & \mathbf{1}_{n}{ }^{T} \delta_{s m U_{-} s k U} & \cdots & \mathbf{1}_{n}{ }^{T} \delta_{s N D_{-} s k D}
\end{array}\right], \\
& \delta_{\text {smu } / D_{-} s k U / D}=\left\{\begin{array}{c}
1 ; m=k \\
0 ; \text { otherwise }
\end{array} .\right. \\
& {\left[\begin{array}{c}
\boldsymbol{V}_{s 1 U} \\
\boldsymbol{V}_{s 1 D} \\
\vdots \\
\boldsymbol{V}_{s k U} \\
\vdots \\
\boldsymbol{V}_{s N D}
\end{array}\right]=\left[\begin{array}{ccccccc}
\boldsymbol{F}_{s 1 U} \boldsymbol{X}^{-1} \boldsymbol{F}_{s 1 U^{T}} & \boldsymbol{F}_{s 1 U} \boldsymbol{X}^{-1} \boldsymbol{F}_{s 1 D}{ }^{T} & \cdots & \boldsymbol{F}_{s 1 U} \boldsymbol{X}^{-1} \boldsymbol{F}_{s k U}{ }^{T} & \cdots & \boldsymbol{F}_{s 1 U} \boldsymbol{X}^{-1} \boldsymbol{F}_{s N D}{ }^{T} \\
\boldsymbol{F}_{s 1 D} \boldsymbol{X}^{-1} \boldsymbol{F}_{s 1 U} & \boldsymbol{F}_{s 1 D} \boldsymbol{X}^{-1} \boldsymbol{F}_{s 1 D}{ }^{T} & \cdots & \boldsymbol{F}_{s 1 D} \boldsymbol{X}^{-1} \boldsymbol{F}_{s k U} U^{T} & \cdots & \boldsymbol{F}_{s 1 D} \boldsymbol{X}^{-1} \boldsymbol{F}_{s N D} \\
\vdots & \vdots & \ddots & \vdots & \ddots & \vdots \\
\boldsymbol{F}_{s k U} \boldsymbol{X}^{-1} \boldsymbol{F}_{s 1 U}{ }^{T} & \boldsymbol{F}_{s k U} \boldsymbol{X}^{-1} \boldsymbol{F}_{s 1 D}{ }^{T} & \cdots & \boldsymbol{F}_{s k U} \boldsymbol{X}^{-1} \boldsymbol{F}_{s k U}{ }^{T} & \cdots & \boldsymbol{F}_{s k U} \boldsymbol{X}^{-1} \boldsymbol{F}_{s N D} \\
\vdots & \vdots & \ddots & \vdots & \ddots & \vdots \\
\boldsymbol{F}_{s N D} \boldsymbol{X}^{-1} \boldsymbol{F}_{s 1 U^{T}} & \boldsymbol{F}_{s N D} \boldsymbol{X}^{-1} \boldsymbol{F}_{s 1 D}{ }^{T} & \cdots & \boldsymbol{F}_{s N D} \boldsymbol{X}^{-1} \boldsymbol{F}_{s k U}{ }^{T} & \cdots & \boldsymbol{F}_{s N D} \boldsymbol{X}^{-1} \boldsymbol{F}_{s N D}{ }^{T}
\end{array}\right]\left[\begin{array}{c}
\boldsymbol{I}_{s 1 U} \\
\boldsymbol{I}_{s 1 D} \\
\vdots \\
\boldsymbol{I}_{s k U} \\
\vdots \\
\boldsymbol{I}_{s N D}
\end{array}\right]}
\end{aligned}
$$

From the equivalent circuit, the voltage of the $k^{\text {th }}$ loop, $V_{s k}$, is the sum of the voltage of the $s k U^{\text {th }}$ loop, $V_{s k U}$, and the voltage of the $s k D^{\text {th }}$ loop, $V_{s k D}$, and is expressed as follows:

$$
V_{s k}=V_{s k U}+V_{s k D}
$$

where

$$
V_{s k U}=\left[\begin{array}{lllll}
v_{s k U .1} & \cdots & v_{s k U . f} & \cdots & v_{s k U . n}
\end{array}\right], \quad V_{s k D}=\left[\begin{array}{lllll}
v_{s k D .1} & \cdots & v_{s k D . f} & \cdots & v_{s k D . n}
\end{array}\right] .
$$

If a current of $1 \mathrm{~A}$ flows through the coil, the total voltage across the coil equals the coil impedance. Therefore, the resistance, $R$, and inductance, $L$, of the coil can be written as follows:

$$
\begin{gathered}
R=\operatorname{Re}\left(\sum_{k=1}^{N}\left(V_{s k U}+V_{s k D}\right)\right), \\
L=\frac{1}{\omega} \operatorname{Im}\left(\sum_{k=1}^{N}\left(V_{s k U}+V_{s k D}\right)\right) .
\end{gathered}
$$

$\operatorname{Re}(\cdot)$ and $\operatorname{Im}(\cdot)$ are the real and imaginary parts of $(\cdot)$, respectively.

\subsection{Calculation Results and Analysis}

For the verification of the proposed method, the calculation results were compared with the results of the simulation using ANSYS Maxwell. For calculation, the multilayer planar spiral coils are considered to be equivalent to the concentric multiloop coil, as shown 
in Figure 2, and $t=2 \mathrm{oz}(\sim 70 \mu \mathrm{m}), T=0.6 \mathrm{~mm}$, and $w 0=0.15 \mathrm{~mm}$ are used. In Figure 5, the change in resistance and inductance with $n$ are compared with the simulation results for double-layer and four-layer planar spiral coils with $g=0.15 \mathrm{~mm}$. The calculation results are in good agreement with the simulation. In Figure $5 c$, the Q-factor of the calculation and simulation are displayed for the four-layer planar spiral coil. The Q-factor is obtained using the resistance and inductance of the coils as follows, where $Q$-factor $=\omega L / R$. $R$ and $L$ are used in Figure $5 \mathrm{~b}$. It should be noted that the optimal $n$ can be determined for the maximum Q-factor because as $n$ increases up to $n=4$, the increase ratio of inductance is higher than that of resistance. In addition, it should be noted that for $N$ greater than or equal to 5 , the increase ratio of inductance reduces although that of resistance continues to increase, because the proximity effect rapidly increases; a similar trend is observed in the case of planar square coils [6]. The inductance and resistance of the four-layer planar spiral coil are more than double those of the double-layer planar spiral coil.

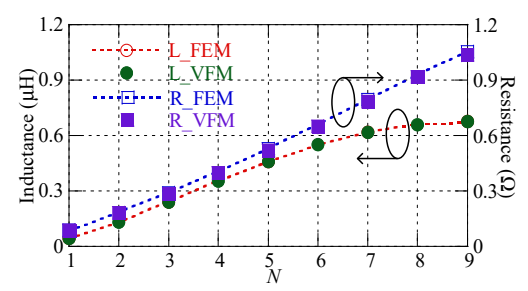

(a)

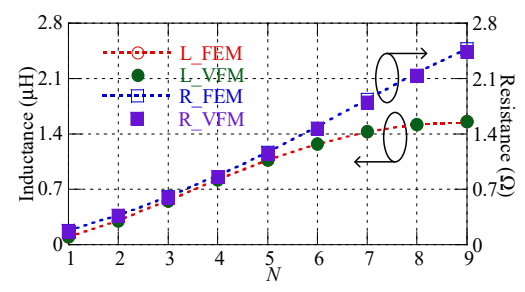

(b)

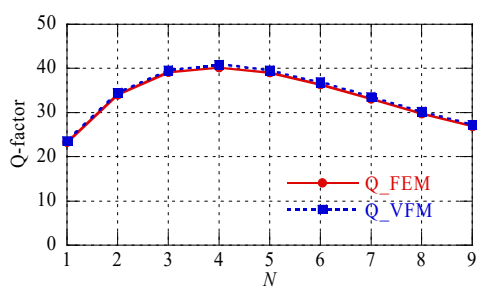

(c)

Figure 5. The calculations and simulations of resistance (R) and inductance (L) according to $N$ for (a) double-layer and (b) four-layer planar spiral coils with $g=0.15 \mathrm{~mm}$. (c) Q-factor for the double-layer planar spiral coil.

In Figure 6, the calculations of resistance and inductance according to the gap $g$ between two neighboring patterns are compared with simulated values for double-layer and four-layer planar spiral coils for $N=4$. As shown, the calculation is in good agreement with the simulation. It is clear that the resistance and inductance decrease with increasing $g$. In Figure 6c, the calculation and simulation of Q-factor are displayed for four-layer planar spiral coils. The optimal gap dimension can be determined for the maximum Q-factor because, as $g$ increases up to $g=0.15 \mathrm{~mm}$, the increase ratio of inductance is higher than that of resistance. The inductance and resistance of the four-layer planar spiral coil are more than double those of double-layer planar spiral coil.

Figure 7 shows the calculated Q-factor according to $g$ and $N$ for the double-layer planar spiral coil with various $w 0$. The optimal $N$ and $g$ for each $w 0$ can be obtained for the maximum Q-factor. The maximum Q-factor is $39.5(L=939.42 \mathrm{nH}, R=1013.37 \mathrm{~m} \Omega)$ for $w 0=100 \mu \mathrm{m}, N=7$, and $g=100 \mu \mathrm{m}$. From the color bar, dark red represents a higher Q-factor. In the area of dark blue, coils cannot be made with the coil dimension.

Figure 8 shows the calculated Q-factors according to $g$ and $N$ for the four-layer planar spiral coil. The maximum Q-factor is $42.5(L=1802.8 \mathrm{nH}, R=1811.5 \mathrm{~m} \Omega)$ for $w 0=100 \mu \mathrm{m}$, $N=6$, and $g=100 \mu \mathrm{m}$. In addition, the maximum Q-factors for $w 0=150 \mu \mathrm{m}, w 0=200 \mu \mathrm{m}$, and $w 0=250 \mu \mathrm{m}$ are $40.8(L=896.8 \mathrm{nH}, R=935.4 \mathrm{~m} \Omega), 40.6(L=500.89 \mathrm{nH}, R=526.16 \mathrm{~m} \Omega)$, and $39.8(L=258.52 \mathrm{nH}, R=276.45 \mathrm{~m} \Omega)$, respectively. It is noted that as $w 0$ increases, the optimal $N$ and coil inductance for the maximum $Q$-factor decrease, whereas the maximum Q-factors are similar regardless of $w 0$. 


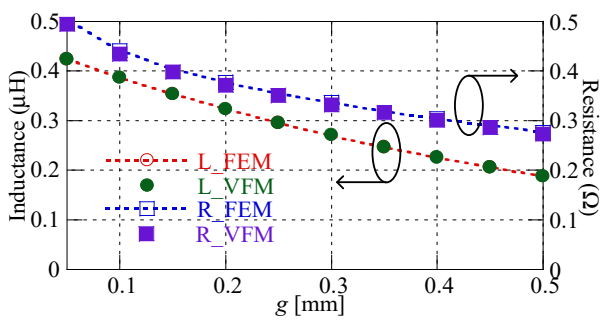

(a)

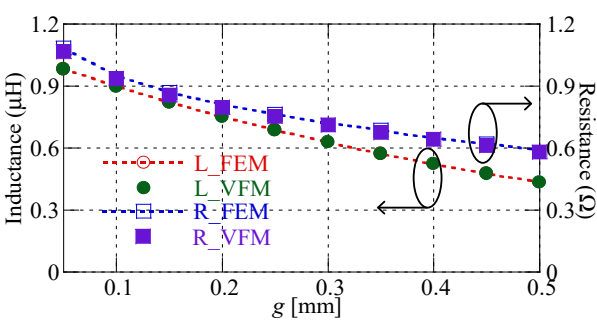

(b)

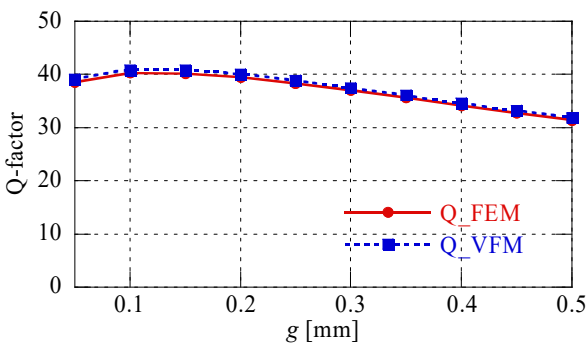

(c)

Figure 6. The calculations and simulations of resistance and inductance according to $g$ for (a) doublelayer and (b) four-layer planar spiral coils with $N=4$. (c) Q-factor for the four-layer planar spiral coil.
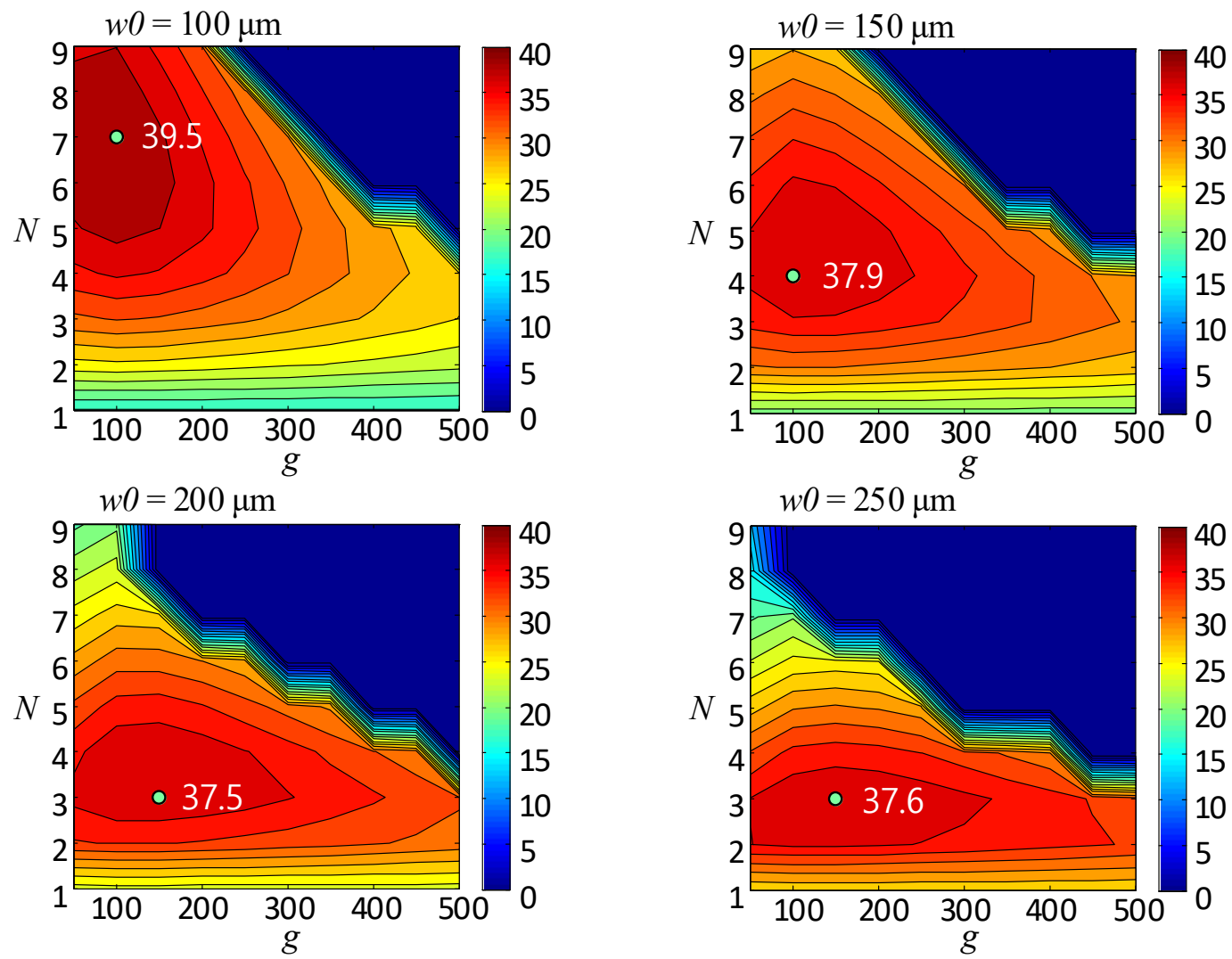

Figure 7. The calculated Q-factor according to $g$ and $N$ for the double-layer planar spiral coil with $w 0=0.1,0.15,0.2$, and $0.25 \mathrm{~mm}$.

Figure 9 shows the calculated Q-factors according to $g$ and $N$ for the eight-layer planar spiral coil. The maximum Q-factor is $51.4(L=2428.0 \mathrm{nH}, R=2.01 \Omega)$ for $w 0=100 \mu \mathrm{m}$, $N=3$, and $g=100 \mu \mathrm{m}$. 

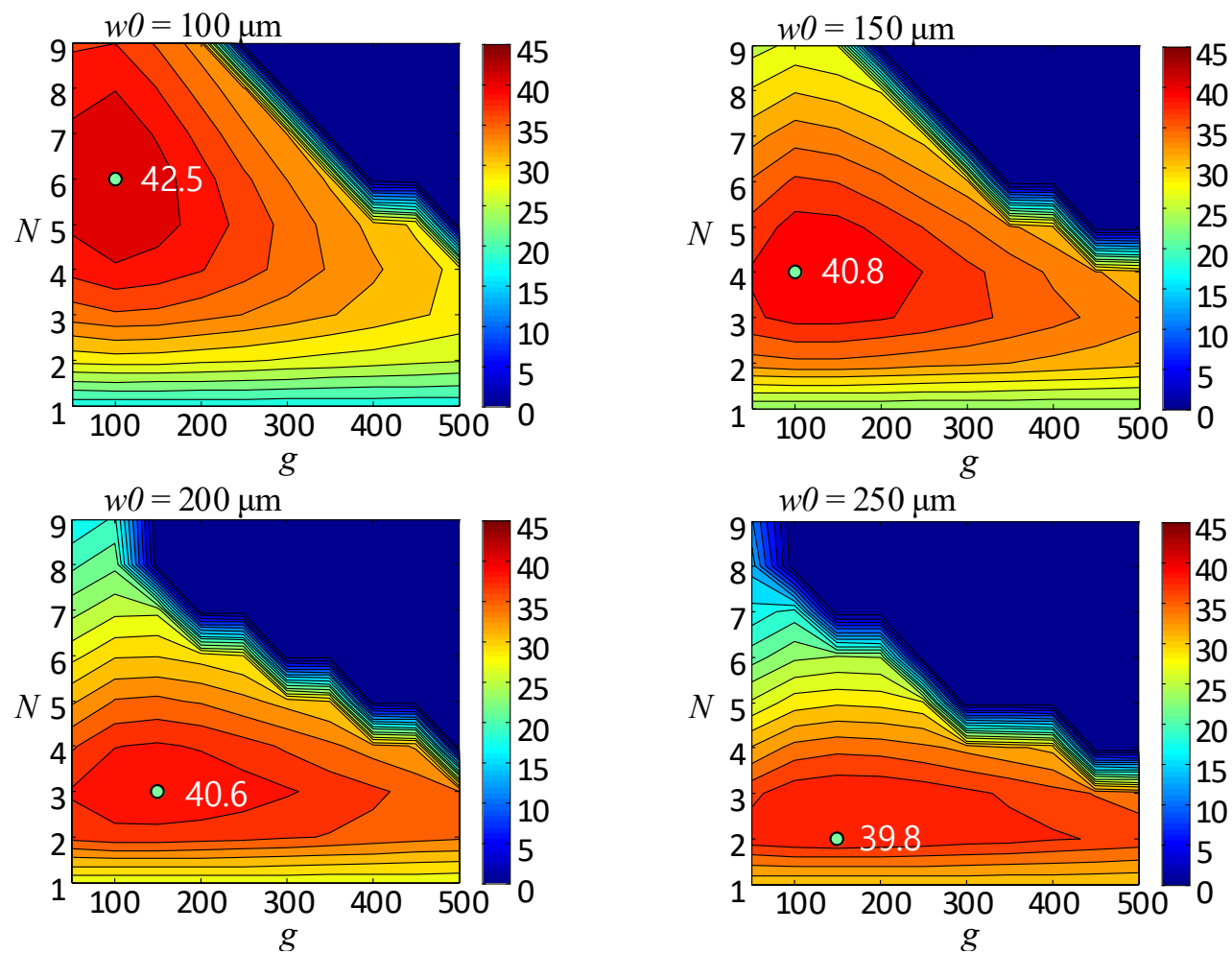

Figure 8. The calculated Q-factor according to $g$ and $N$ for the four-layer planar spiral coil for $w 0=0.1,0.15,0.2$, and $0.25 \mathrm{~mm}$.
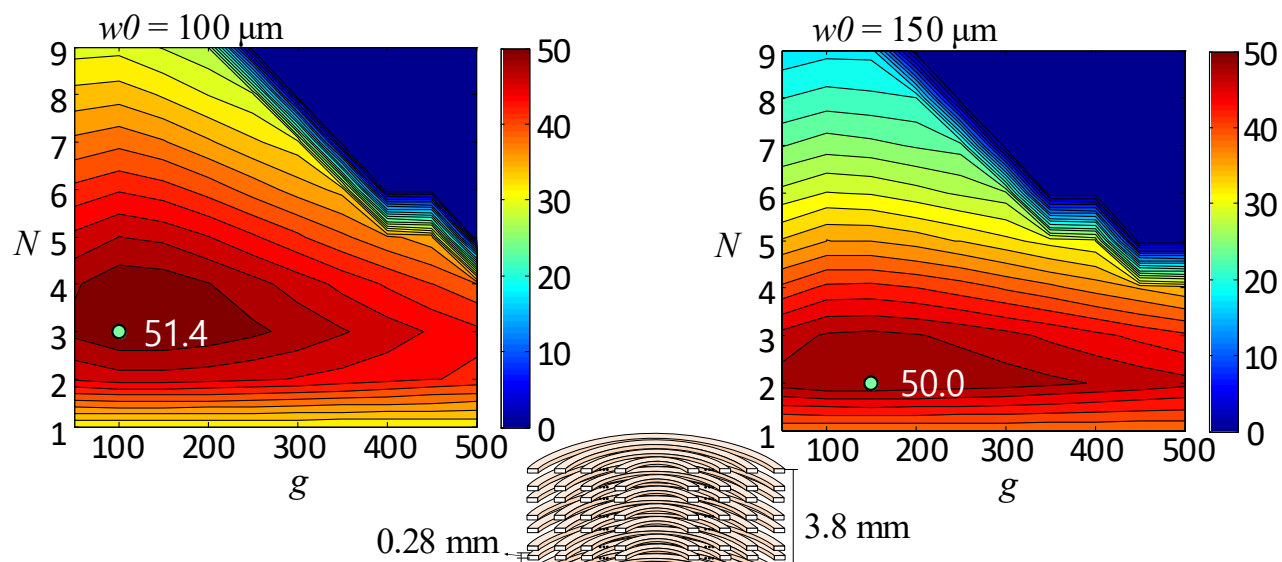

$3.8 \mathrm{~mm}$
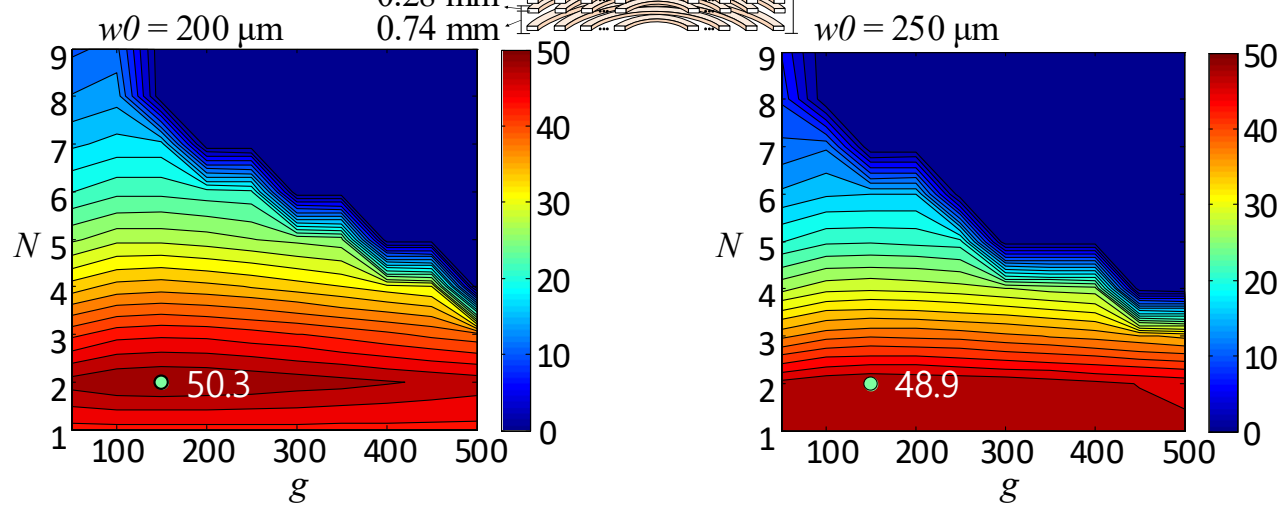

Figure 9. The calculated $Q$-factor according to $g$ and $N$ for the eight-layer planar spiral coil for $w 0=0.1,0.15,0.2$, and $0.25 \mathrm{~mm}$. 
From Figures 7-9, the eight-layer planar spiral coil has the highest maximum Q-factor, compared with the double- and four-layer planar spiral coils. This is because the increase in the inductance is higher than that of the resistance at optimal $N$ and $g$ with the increase in the number of coil layers. As the number of coil layers increases up to eight layers, the maximum Q-factors increase, whereas the optimal turn number decreases. In addition, it is shown that the maximum Q-factors are similar regardless of $w 0$ for each layer, whereas the optimal dimensions for the maximum Q-factor vary in relation to $w 0$.

Because the optimal dimensions required for the maximum $Q$-factor may differ from those required for the maximum coupling efficiency, the maximum coupling efficiency according to $g$ and $N$ for double-, four-, and eight-layer planar spiral coils with various $w 0$ are calculated. The maximum coupling efficiency, $\eta$, can be calculated as follows [14,15]:

$$
\eta=\frac{\text { FoM }^{2}}{\left(\sqrt{1+\text { FoM }^{2}}+1\right)^{2}}=\frac{\sqrt{1+\text { FoM }^{2}}-1}{\sqrt{1+\text { FoM }^{2}}+1} \text { with FoM }=\frac{\omega \mathrm{M}_{\mathrm{Tx}}-\mathrm{Rx}}{\sqrt{\mathrm{R}_{\mathrm{Tx}} \cdot \mathrm{R}_{\mathrm{Rx}}}} .
$$

$R_{T x}$ and $R_{R x}$ are the resistances of the Tx and Rx coils, respectively. $M_{T x-R x}$ is the mutual inductance between the Tx and Rx coils. FoM stands for the Figure of Merit of the MR-WPT system.

In Figure 10, the cross-sectional and top views of the Tx coil and four-layer Rx coil for calculation are shown. The Rx coil is placed in the middle of Tx coil, and the bottom of the $\mathrm{Rx}$ coil is placed slightly higher than $\mathrm{z}=1.2 \mathrm{~mm}$ because a practical bowl will have a finite thickness of about $1 \mathrm{~mm}$.

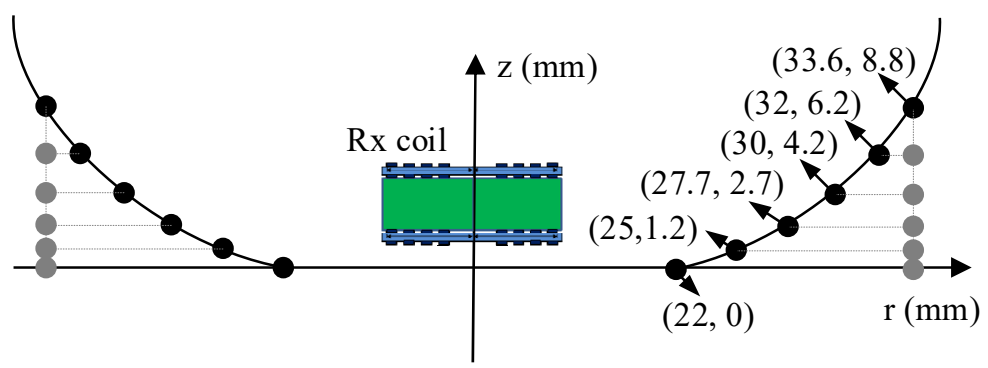

(a)

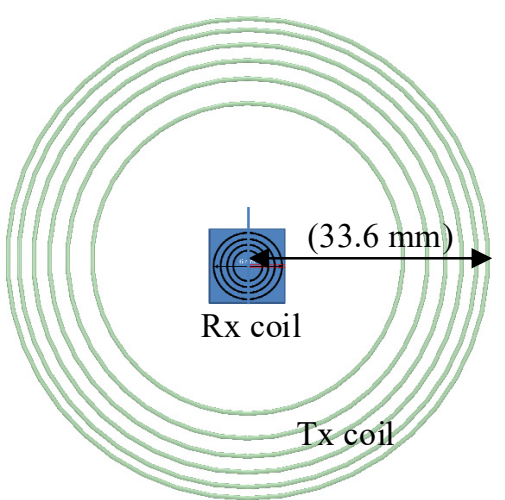

(b)

Figure 10. (a) The cross-sectional and (b) top views of the glass-shaped Tx coil and four-layer Rx coil for calculation. In the cross-sectional view, a helical coil is also displayed (gray color).

As shown in Figure 10, the bowl-shaped coil of six turns was designed by referring to [16]. It is advantageous to obtain a smaller skin resistance when the diameter of the copper wire is larger than the skin depth of $6.78 \mathrm{MHz}$, whereas the bowl-shaped coil has a higher magnetic field than the helix coil. The four-turn coils have lower magnetic fields than the six-turn coils.

For a high and uniform magnetic field between $\mathrm{z}=1.5 \mathrm{~mm}$ and $\mathrm{z}=6 \mathrm{~mm}$ in the bowl, the Tx coil was designed. It can be noted that a four-layer $\mathrm{Rx}$ coil will be placed between $\mathrm{z}=1.5 \mathrm{~mm}$ and $\mathrm{z}=6 \mathrm{~mm}$ in the bowl. By using the calculation equations in [16], for a $\mathrm{z}$-directed magnetic field, $\mathrm{H}_{\mathrm{z}}$ is calculated. In Figure $11 \mathrm{a}$, the calculated magnetic fields at $\mathrm{z}=1.5$ and Figure $11 \mathrm{~b} \mathrm{z}=6 \mathrm{~mm}$ are displayed. In addition, the calculation results of helix coils are compared with the bowl-shaped coils for four- and six-turn coils. For the case of four turns, two turns from the top are removed. Both Tx and helix coils of six turns for $\mathrm{z}=1.5 \mathrm{~mm}$ and $6 \mathrm{~mm}$ have a uniform magnetic field in the charging area from $r=0$ to $r=20 \mathrm{~mm}$, whereas the bowl-shaped coil has a higher magnetic field than the helix coil. The four-turn coils have lower magnetic fields than the six-turn coils. 


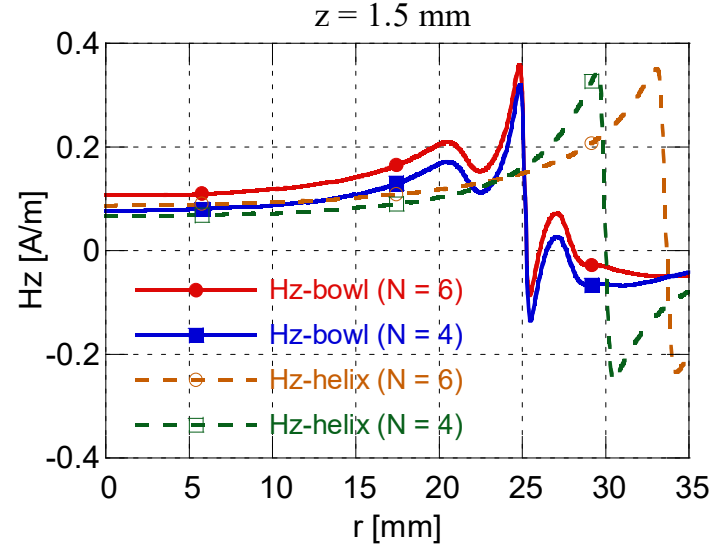

(a)

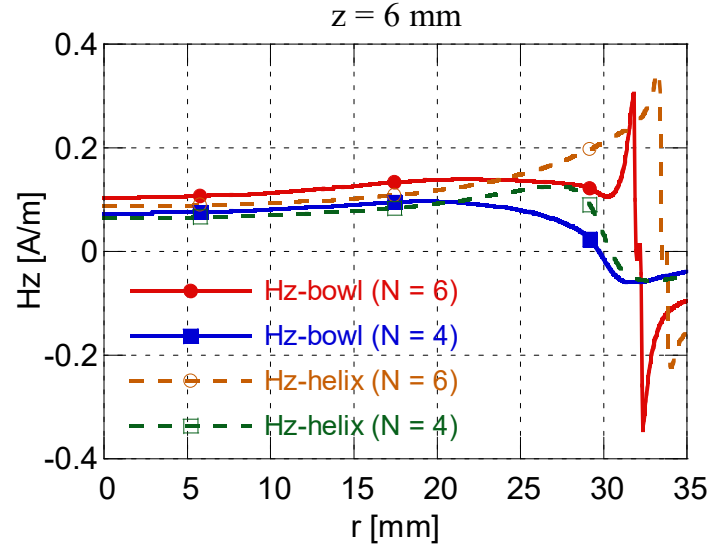

(b)

Figure 11. Calculated magnetic fields at (a) $\mathrm{z}=1.5 \mathrm{~mm}$ and $(\mathbf{b}) \mathrm{z}=6 \mathrm{~mm}$ for the bowl-shaped Tx coil and helix coils of fourand six-turn coils.

Figures 12-14 show the calculated maximum coupling efficiency (\%) according to $g$ and $N$ for double-layer, four-layer, and eight-layer planar spiral coils with $w 0=100,150$, 200, and $250 \mu \mathrm{m}$. As shown, the optimal $N$ and $g$ for each $w 0$ was found to obtain the maximum coupling efficiency. The dimensions for the maximum coupling efficiency are similar to those for the maximum Q-factor, except for those for $w 0=100 \mu \mathrm{m}$. Further, the maximum coupling efficiency increases with more layers, up to eight layers, whereas maximum coupling efficiencies for each layer are similar regardless of $w 0$. It is noted that optimal coil dimensions for each layer should be found for maximum coupling efficiency.
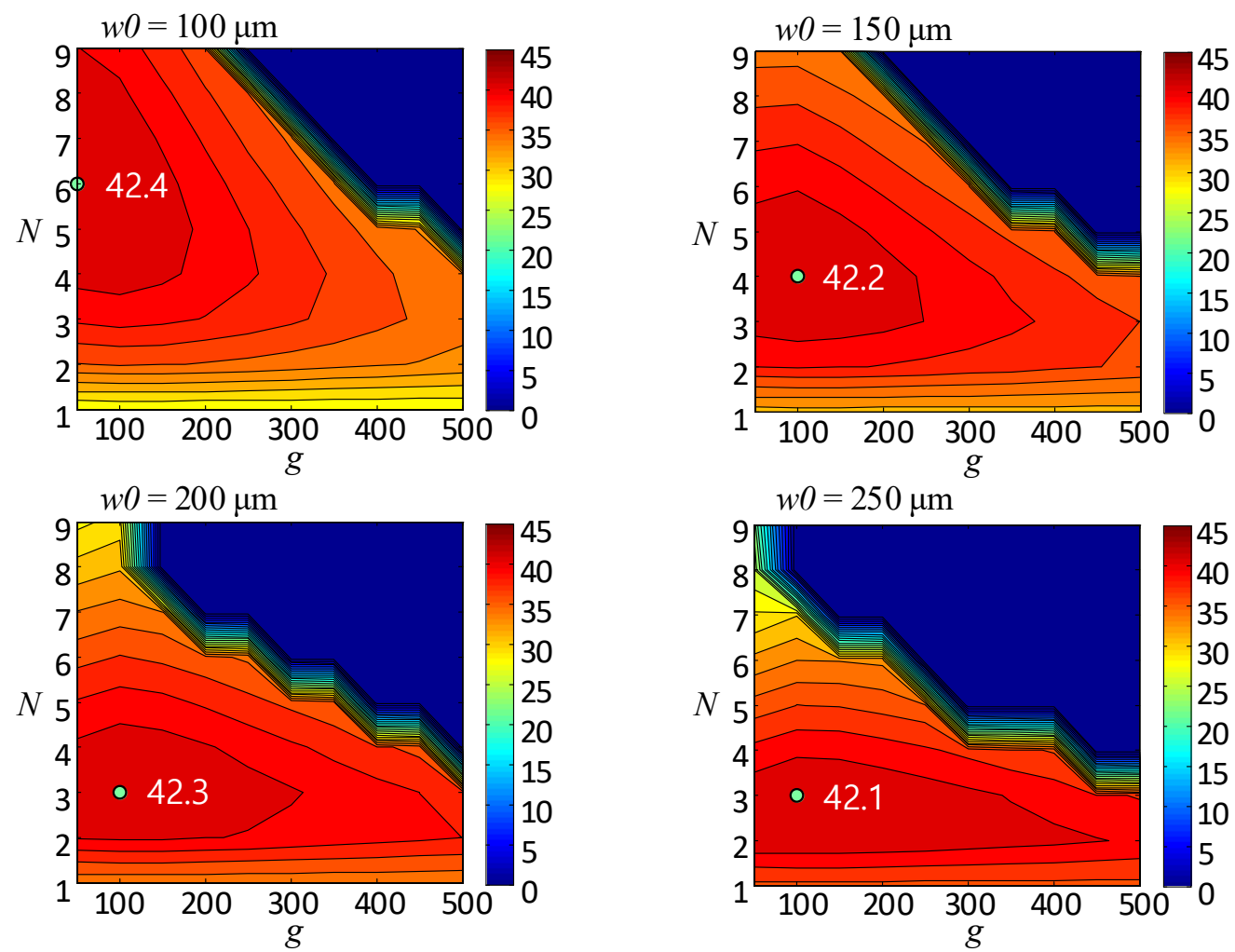

Figure 12. The calculated maximum coupling efficiency (\%) according to $g$ and $N$ for the double-layer planar spiral coils for $w 0=100,150,200$, and $250 \mu \mathrm{m}$. 

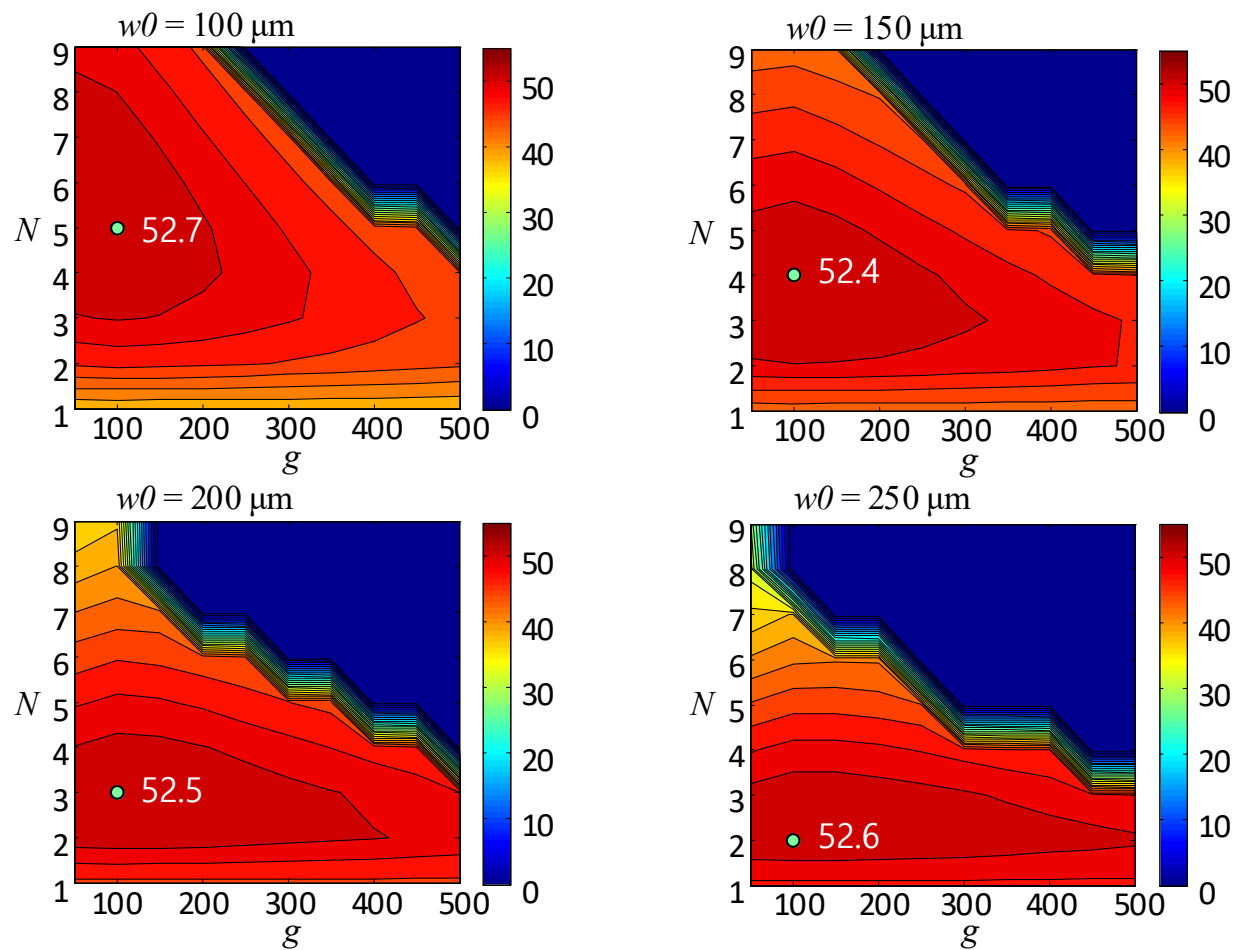

Figure 13. The calculated maximum coupling efficiency (\%) according to $g$ and $N$ for the four-layer planar spiral coil for $w 0=100,150,200$, and $250 \mu \mathrm{m}$.
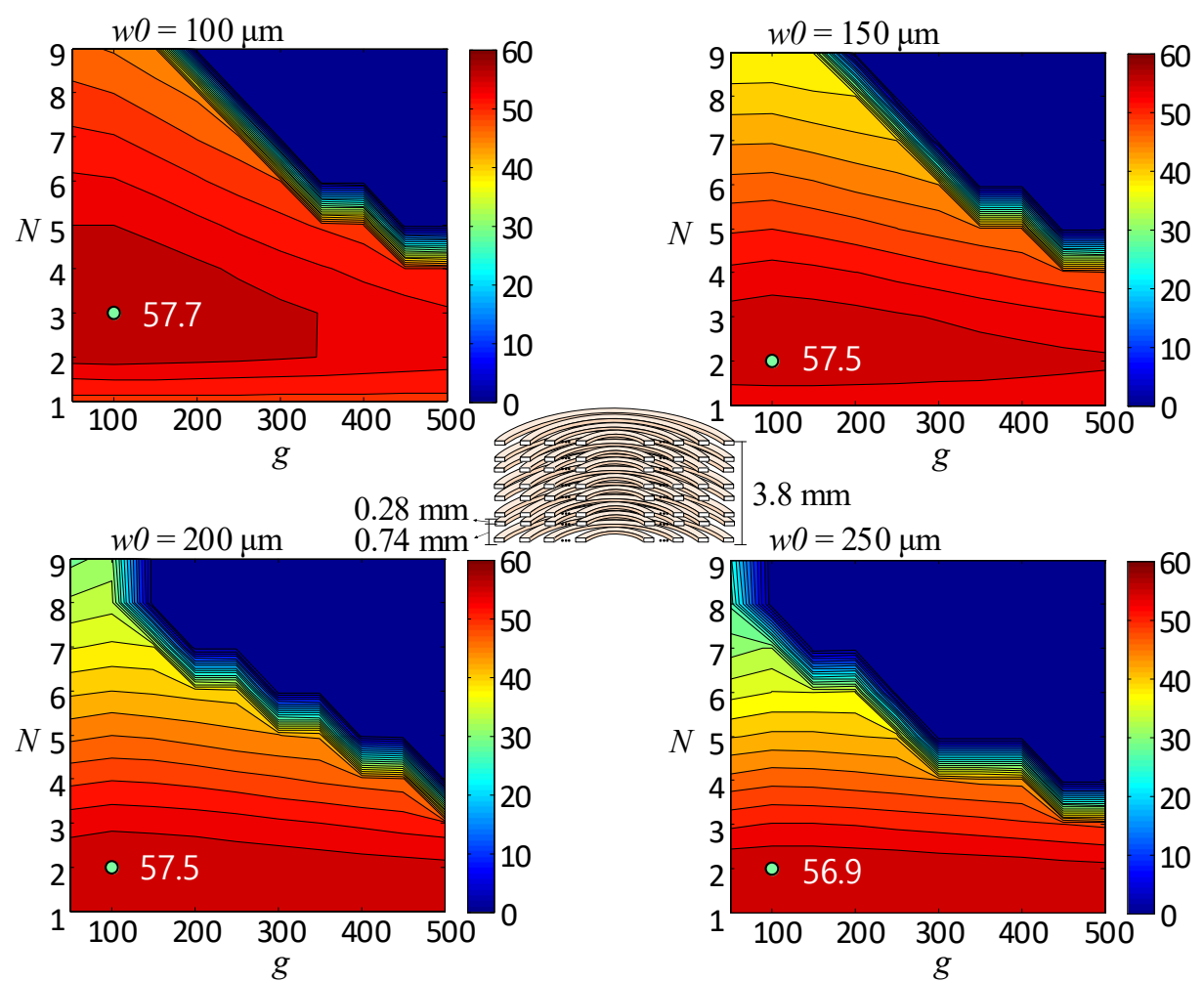

$3.8 \mathrm{~mm}$

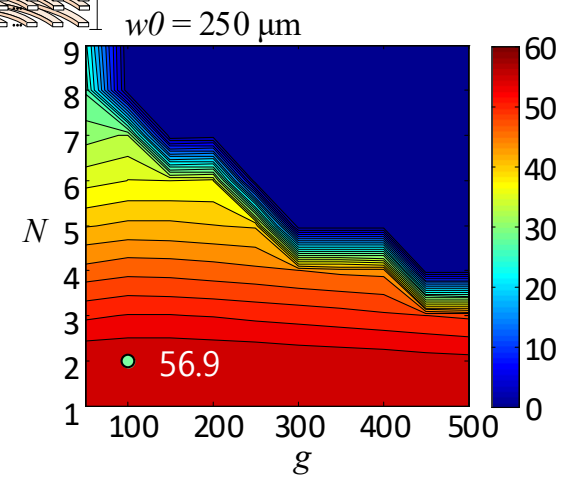

Figure 14. The calculated maximum coupling efficiency (\%) according to $g$ and $N$ for the eight-layer planar spiral coil for $w 0=100,150,200$, and $250 \mu \mathrm{m}$.

It is shown that the eight-layer planar spiral coil has the highest maximum coupling efficiency. This is because the Q-factor for the eight-layer coil is the highest of the double-, 
four-, and eight-layer coils, as shown in Figures 7-9. Additionally, the mutual inductance between the Tx coil and Rx coil for the eight-layer planar spiral coil is the highest.

Based on the findings from Figures $12-14, N=4, w 0=150 \mu \mathrm{m}$, and $g=200 \mu \mathrm{m}$ for a four-layer planar spiral coil were chosen as the optimal values for the device by considering easy fabrication, reasonable manufacturing cost, and easy integration with the main PCB board. Moreover, from the practical viewpoint, it is more advantageous to design a coil of higher inductance for small WPT systems. That is, for the maximum Q-factor for the four-layer coil, $L=896.8 \mathrm{nH}$ for $w 0=150 \mu \mathrm{m}$ is higher than those of $L=500.89 \mathrm{nH}$ for $w 0=200 \mu \mathrm{m}$ and $L=258.52 \mathrm{nH}$ for $w 0=250 \mu \mathrm{m}$.

\section{Coil Fabrication and Measurement}

A four-layer planar spiral coil was fabricated using the calculated parametric values. The four-layer planar spiral coil was made by connecting two double-layer planar spiral coils in series. Figure 15a shows a top view of a double-layer planar spiral coil. The coil specification is $N=4, w 0=150 \mu \mathrm{m}, g=200 \mu \mathrm{m}, t=2 \mathrm{oz}$, radius of outmost loop $R R=3.0 \mathrm{~mm}$, and $T=0.6 \mathrm{~mm}$. In Figure $15 \mathrm{~b}$, the fabricated four-layer planar spiral coil is displayed. The distance between two double-layer planar spiral coils is $2.3 \mathrm{~mm}$. Figure $15 \mathrm{c}$ shows the PCB of the bio-signal monitoring device. Figure 15d shows the back view of the circuit board of the four-layer planar spiral coil.

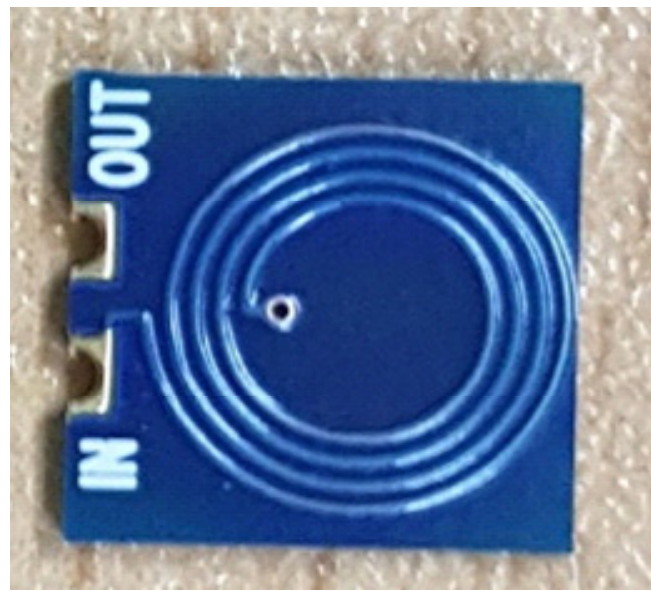

(a)

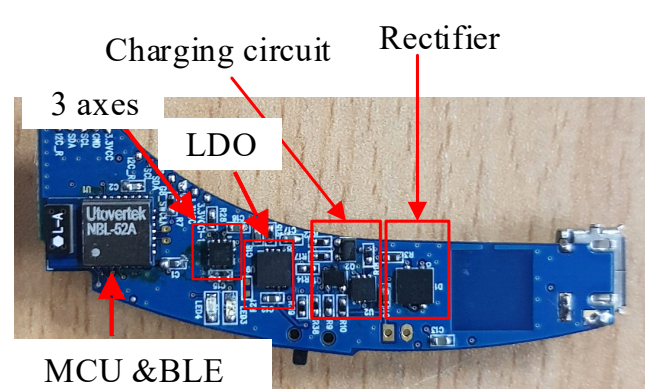

(c)

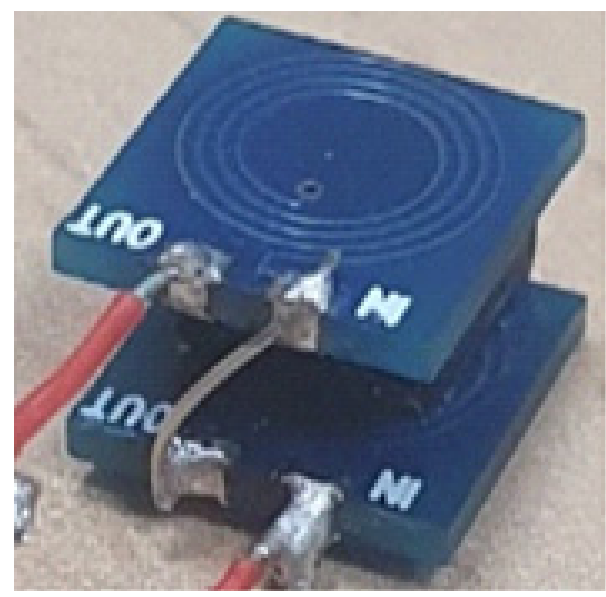

(b)

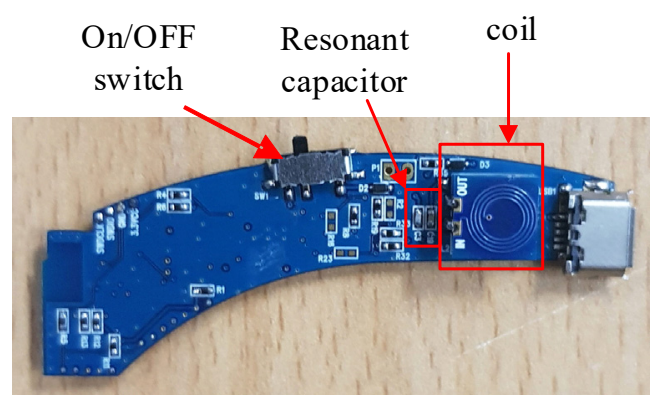

(d)

Figure 15. (a) Photographs of the fabricated double-layer planar spiral coil; (b) four-layer planar spiral coil; (c) circuit board of the bio-signal monitoring device;(d) back view of the circuit board of the four-layer planar spiral coil.

Figure 16 shows the measurement setup. Rx1 is a double-layer planar spiral coil as shown in Figure 15a. Rx2, shown in Figure 15b, is a four-layer planar spiral coil. Position 1 is in the middle of the Tx coil and position 2 is at the border of the glass. Rx3 is a four-layer planar spiral coil on the Rx board. For the measurement of resistance, inductance, and mutual inductance, the Agilent E5072A network analyzer was used. 

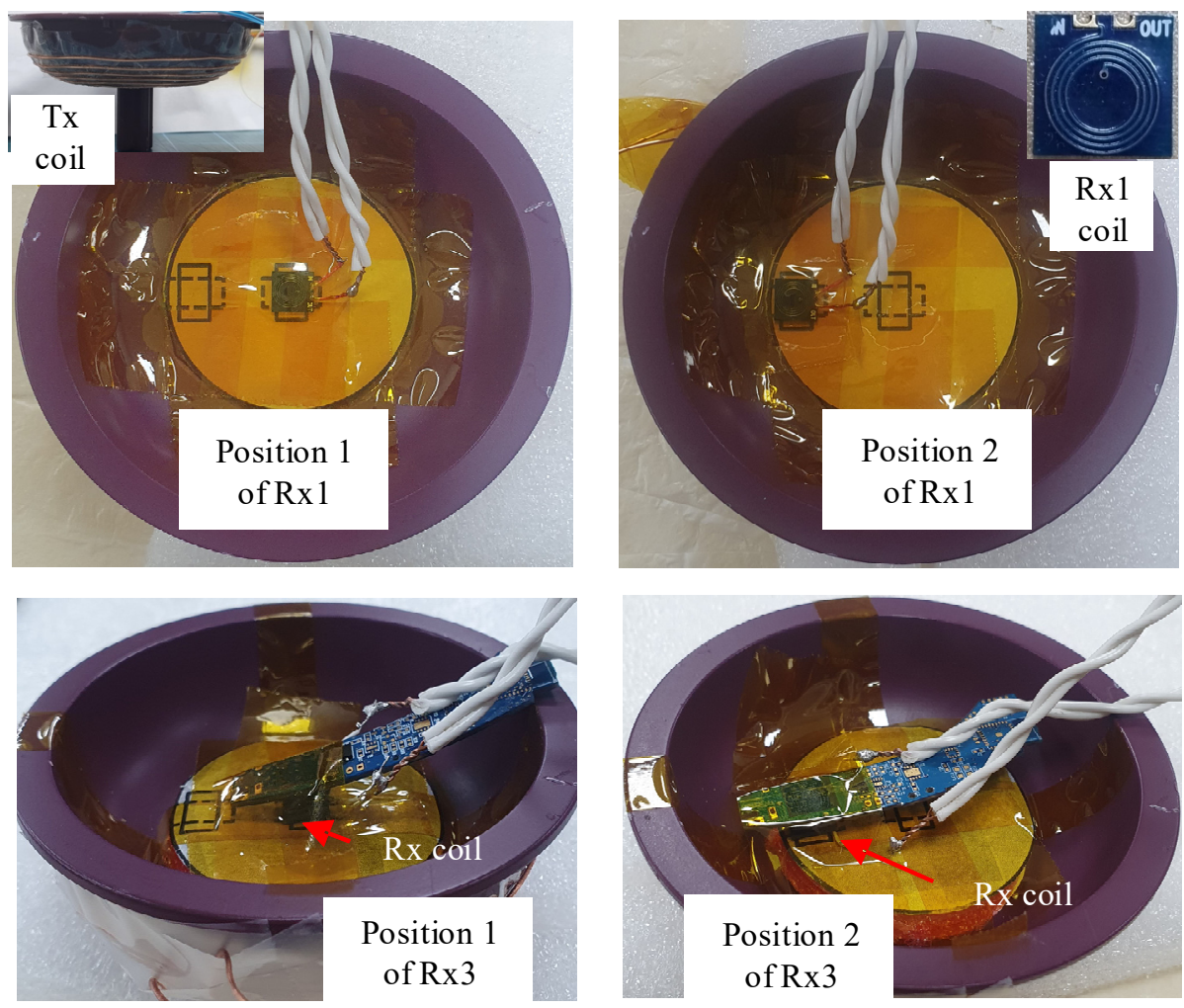

Figure 16. The measurement setups of the Rx coils without (upper) and with (lower) an Rx board.

The fabricated Tx coil is shown in the inset of the upper-left figure shown in Figure 16. The Tx coil was also measured, and the inductance and AC resistance were $3.0322 \mu \mathrm{H}$ and $429.15 \mathrm{~m} \Omega$, respectively.

Table 1 lists the measurement results, with the calculation results for comparison. Inductance, resistance, and mutual inductance measurements are shown in the table. The measurements of FoM and maximum coupling efficiency in the table were obtained using Equation (15), and the resistance, inductance, and mutual inductance were measured. The measured resistance and inductance are in good agreement with the calculated values. However, the resistance values slightly differ because the coil pattern of the fabricated device is slightly narrower for $w 0=130 \mu \mathrm{m}$, in comparison with the designed value of $150 \mu \mathrm{m}$. Moreover, the maximum coupling efficiency is obtained at the edge because the mutual inductance at position 2 is higher than that at position 1, as shown in Table 1.

Table 1. The measured and calculated results for the double-layer planar spiral coil (Rx1), and four-layer planar spiral coil without (Rx2) and with a device board (Rx3).

\begin{tabular}{|c|c|c|c|c|c|c|c|c|c|c|c|}
\hline & \multirow[b]{2}{*}{ Position } & \multicolumn{5}{|c|}{ Measurement } & \multicolumn{5}{|c|}{ Calculation } \\
\hline & & $\mathrm{L}[\mathrm{nH}]$ & $\mathrm{R}[\mathrm{m} \Omega]$ & $\mathbf{M}[\mathrm{nH}]$ & FoM & $\begin{array}{c}\text { Maximum } \\
\text { Coupling } \\
\text { Efficiency } \\
(\%)\end{array}$ & $\mathrm{L}[\mathrm{nH}]$ & $\mathrm{R}[\mathrm{m} \Omega]$ & $\mathrm{M}[\mathrm{nH}]$ & FoM & $\begin{array}{c}\text { Maximum } \\
\text { Coupling } \\
\text { Efficiency } \\
(\%)\end{array}$ \\
\hline \multirow{2}{*}{$\mathrm{Rx} 1$} & 1 & 307.31 & 444.29 & 21.5 & 2.10 & 39.9 & 323.20 & 372.03 & 19.9 & 2.18 & 41.1 \\
\hline & 2 & 307.31 & 444.29 & 32.7 & 3.19 & 54.0 & 323.20 & 372.03 & 27.4 & 3.01 & 52.1 \\
\hline \multirow{2}{*}{$\mathrm{R} \times 2$} & 1 & 695.06 & 919.88 & 36.1 & 2.45 & 45.1 & 750.57 & 798.96 & 39.4 & 2.95 & 51.4 \\
\hline & 2 & 695.06 & 919.88 & 53.7 & 3.64 & 58.1 & 750.57 & 798.96 & 51.9 & 3.89 & 60.1 \\
\hline \multirow{2}{*}{$\mathrm{R} \times 3$} & 1 & 693.02 & 929.24 & 43.0 & 2.90 & 50.8 & - & - & - & - & - \\
\hline & 2 & 693.02 & 929.24 & 61.5 & 4.15 & 62.0 & - & - & - & - & - \\
\hline $\mathrm{Tx}$ & & 3032.2 & 429.15 & & & & 2777.0 & 404.39 & & & \\
\hline
\end{tabular}




\section{Wearable Bio-Signal Monitoring Device and Wireless Charging}

Figure 17 shows a block diagram of the proposed MR-WPT system for biomedical devices. For the Tx system, the Tx circuit in [17] was used.

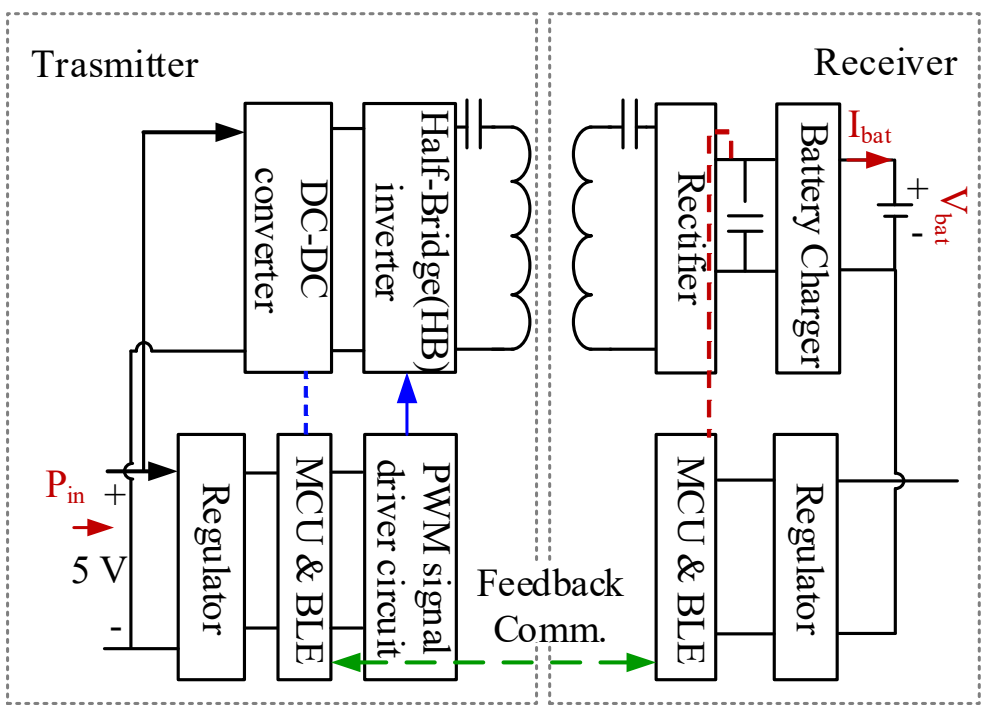

Figure 17. Block diagram of the proposed MR-WPT system for biomedical devices.

In Figure 18, a block diagram of a bio-signal monitoring device and wireless charging station are shown. The device comprises a wireless charger, BLE for the communication of the sensor data, a wireless charging control station, a three axis sensor, a skin temperature sensor (in-ear), a geographic information systems (GIS) sensor, and a photoplethysmography (PPG) sensor (in ear). The wireless charger is designed with two power stages, which consist of a full bridge rectifier (SBR05M100BLP), charger IC (MCP73830L), and LDO (LT3802EDD) to minimize the power conversion efficiency.

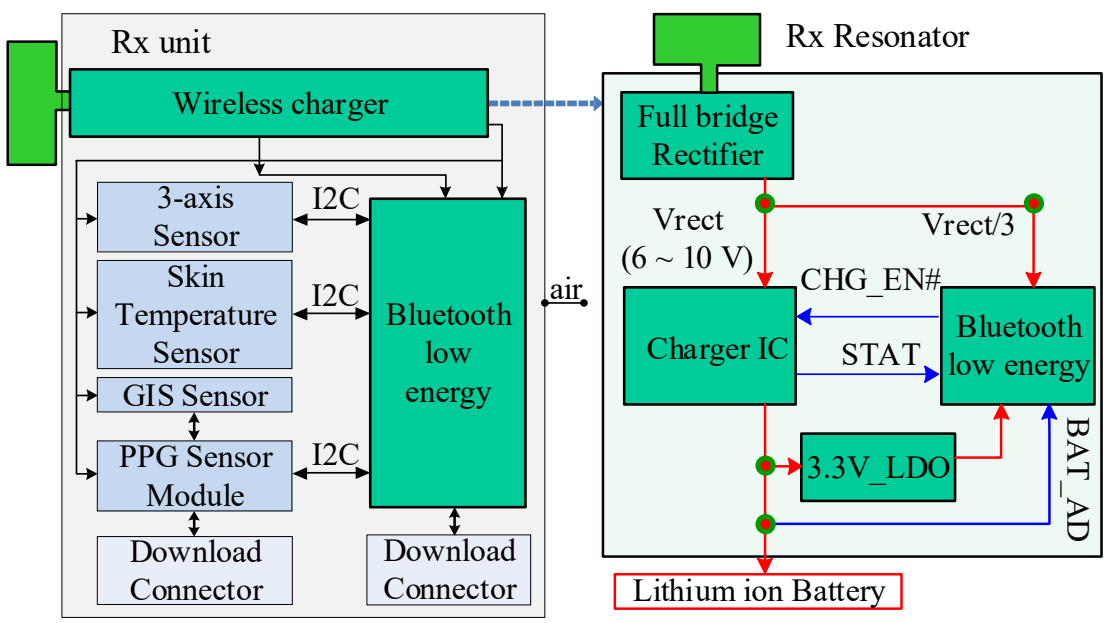

Figure 18. Block diagram of a bio-signal monitoring device and wireless charging block.

Figure 19 shows the experimental setup. A KEITHLEY 2280S-60-3 was used for the power supply, and the KEITHLEY 2280S-60-3 as a battery simulator was used for the battery charging experiment. The total power to the Tx board was about $1.3 \mathrm{~W}(5 \mathrm{~V} / 0.26 \mathrm{~A})$, which includes the power consumption for BLE communication. The measured Rx power of the battery simulator was $0.209 \mathrm{~mW}(4.152 \mathrm{~V} / 50.4 \mathrm{~mA})$. Therefore, the wireless power transfer efficiency from the Tx board to Rx battery was $16.08 \%$. The power conversion efficiency 
for the Tx and Rx circuits can be improved, especially for the low-power applications of wearable devices.

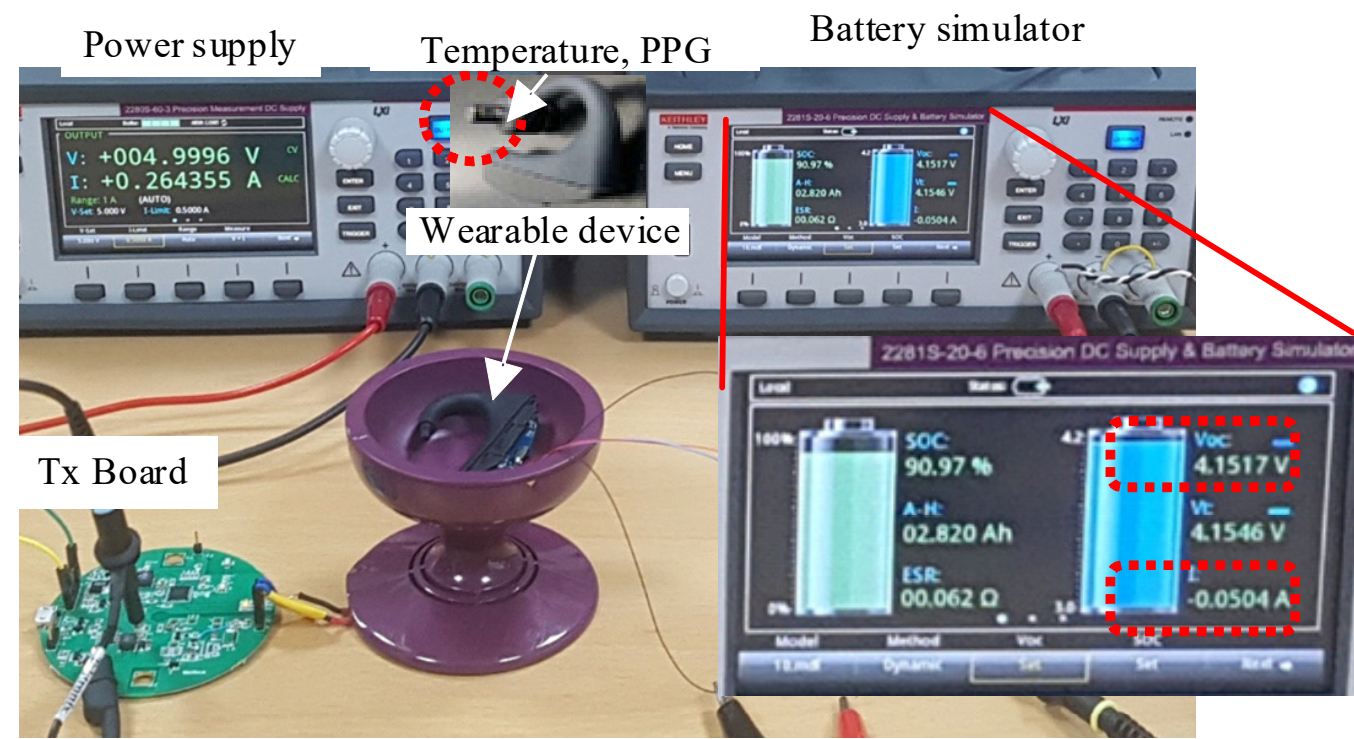

Figure 19. The experimental setup for the wireless power transfer system for a wearable bio-signal monitoring device.

Finally, this work is compared with previous works in Table 2. As shown, the coil in the paper is the smallest multilayer planar spiral coil for MR-WPT applications at $6.78 \mathrm{MHz}$. In addition, the dc-dc efficiency of this work is higher than that in [7], in which a helix Tx coil and a double-layer planar spiral Rx coil were used.

Table 2. Comparison between previous works and this work.

\begin{tabular}{cc|c|c|c}
\hline & {$[7]$} & {$[9]$} & {$[11]$} & This Work \\
\hline $\begin{array}{c}\text { Rx coil size, metal } \\
\text { thickness }\end{array}$ & $\begin{array}{c}15 \mathrm{~mm}(\mathrm{~W}) \times 10 \mathrm{~mm}(\mathrm{~L}), \\
1 \mathrm{oz}\end{array}$ & $\begin{array}{c}288 \mathrm{~mm} \times 288 \mathrm{~mm}, \\
3 \mathrm{oz}\end{array}$ & $\begin{array}{c}64 \mathrm{~mm}(\mathrm{~W}) \times 64 \mathrm{~mm}(\mathrm{~L}), \\
1 \mathrm{oz}\end{array}$ & $\begin{array}{c}6 \mathrm{~mm}(\mathrm{~W}) \times 6 \mathrm{~mm}(\mathrm{~L}), 2 \mathrm{oz} \\
(70 \mu \mathrm{m})\end{array}$ \\
\hline Number of layers & $2(\mathrm{Rx}$ coil $)$ & $2(\mathrm{Rx}$ coil $)$ & $12(\mathrm{Tx}$ coil $)$ & $4(\mathrm{Rx} \mathrm{coil)}$ \\
\hline Frequency $(\mathrm{MHz})$ & 6.78 & 4.03 & 80 & 6.78 \\
\hline Coil-to-coil/ & NA, & NA, & $80 \%$, & $58.1 \%$, \\
dc-dc efficiency & less than $1 \%$ & NA & NA & $16.1 \%$ \\
\hline
\end{tabular}

\section{Conclusions}

This study analyzed multilayer planar spiral coils applied to MR-WPT in the MHz frequency range for use in compact wearable bio-signal monitoring devices using the VFM. In the current paper, the calculation method for the AC resistance and inductance using the VFM is explained in detail. Moreover, the planar spiral coil can be optimized to obtain the maximum Q-factor and maximum coupling efficiency using the calculation. As the number of layers of coils increases, the maximum Q-factor and maximum coupling efficiency increase, up to a total of eight layers, when the coils are optimized using the proposed method. The calculation method was verified via simulations and measurements. It is shown that the proposed method is able to analyze the AC resistance and inductance inclusive of the proximity loss and skin effects in the $\mathrm{MHz}$ frequency range. Finally, a four-layer planar spiral coil was designed using the proposed optimization method to be used for wireless charging of a compact wearable bio-signal monitoring device. To the best of the authors' knowledge, the proposed device is the smallest multilayer planar spiral coil for MR-WPT application at $6.78 \mathrm{MHz}$. In addition, the optimization process 
of multiple layer planar spiral coils is shown for the maximum Q-factor and maximum coupling efficiency. The proposed method is applicable to the optimization of planar spiral coils for use in mobile devices.

Author Contributions: Conceptualization, Y.-J.P.; methodology, Y.-J.P. and J.-E.K.; validation, J.-E.K., K.-M.N., K.-D.Y. and K.-H.C.; formal analysis, Y.-J.P. and J.-E.K.; investigation, Y.-J.P.; resources, Y.-J.P.; data curation, J.-E.K. and K.-M.N.; writing—original draft preparation, Y.-J.P.; writing—review and editing, Y.-J.P. and J.-E.K.; visualization, J.-E.K.; supervision, Y.-J.P.; project administration, Y.-J.P.; funding acquisition, Y.-J.P. All authors have read and agreed to the published version of the manuscript.

Funding: This research was funded by the KERI Primary research program of MSIT/NST, grant number 21A01033 and the Industrial Infrastructure Program for Fundamental Technologies of KIAT, grant number P0014854 (21A02059), and the Ministry of Trade, Industry and Energy (MOTIE), Korea.

Data Availability Statement: Not applicable.

Conflicts of Interest: The authors declare no conflict of interest.

\section{References}

1. Won, S.M.; Song, E.; Reeder, E.T.; Rogers, J.A. Emerging Modalities and Implantable Technologies for Neuromodulation. Cell 2020, 181, 115-135. [CrossRef]

2. Won, S.M.; Cai, L.; Gutruf, P.; Rogers, J.A. Wireless and battery-free technology for neuroengineering. Nat. Biomed. Eng. 2021, 1-19. [CrossRef]

3. Jeong, S.; Kim, D.; Song, J.; Kim, H.; Lee, S.; Song, C.; Lee, J.; Song, J.; Kim, J. Smartwatch strap wireless power transfer system with flexible PCB coil and shielding material. IEEE Trans. Ind. Electron. 2019, 66, 4054-4064. [CrossRef]

4. Wagih, M.; Komolafe, A.; Zaghari, B. Wearable Wireless Power Transfer using Direct-Write Dispenser Printed Flexible Coils. In Proceedings of the 2020 IEEE International Conference on Flexible and Printable Sensors and Systems (FLEPS), Manchester, UK, 16-19 August 2020; pp. 1-4. [CrossRef]

5. Jow, U.-M.; Ghovanloo, M. Design and Optimization of Printed Spiral Coils for Efficient Transcutaneous Inductive Power Transmission. IEEE Trans. Biomed. Circuits Syst. 2007, 1, 193-202. [CrossRef] [PubMed]

6. Kim, D.-H.; Park, Y.-J. Calculation of the inductance and AC resistance of planar rectangular coils. Electron. Lett. 2016, 52, 1321-1323. [CrossRef]

7. Kim, C.Y.; Ku, M.J.; Qazi, R.; Nam, H.J.; Park, J.W.; Nam, K.S.; Oh, S.; Kang, I.; Jang, J.-H.; Kim, W.Y.; et al. Soft subdermal implant capable of wireless battery charging and programmable controls for applications in optogentics. Nat. Commun. 2021, 12, 535. [CrossRef] [PubMed]

8. Lope, I.; Carretero, C.; Acero, J.; Alonso, R.; Burdio, J.M. AC Power Losses Model for Planar Windings With Rectangular Cross-Sectional Conductors. IEEE Trans. Pow. Electron. 2014, 29, 23-28. [CrossRef]

9. Chen, K.; Zhao, Z. Analysis of the Double-Layer Printed Spiral Coil for Wireless Power Transfer. IEEE Trans. Emerg. Sel. Topics Power Electron. 2013, 1, 114-121. [CrossRef]

10. Gong, F.; Cong, Y.; Wei, Z.; Yin, B.; Chi, H.; Luan, X. Analysis of the multi-layer Printed Spiral Coil for wireless power transfer system used in medical implants. 2016 Asia-Pacific Int. Symp. Electromag. Comp. (APEMC) 2016, 1, 460-462. [CrossRef]

11. Bao, K.; Zekios, C.L.; Georgakopoulos, S.V. A wearable WPT system on flexible substrates. IEEE Antennas Wireless Propag. Lett. 2019, 18, 931-935. [CrossRef]

12. Kim, D.-H.; Kim, J.; Park, Y.-J. Optimization and Design of Small Circular Coils in a Magnetically Coupled Wireless Power Transfer System in the Megahertz Frequency. IEEE Trans. Micro. Theory Tech. 2016, 64, 1-12. [CrossRef]

13. Good, R.H. Elliptic integrals, the forgotten functions. Eur. J. Phys. 2001, 22, 119-126. [CrossRef]

14. Kurs, A.; Karalis, A.; Moffatt, R.; Joannopoulos, J.D.; Fisher, P.; Soljacic, M. Wireless Power Transfer via Strongly Coupled Magnetic Resonances. Science 2007, 317, 83-86. [CrossRef]

15. Kim, J.; Kim, D.-H.; Park, Y.-J. Free-Positioning Wireless Power Transfer to Multiple Devices Using a Planar Transmitting Coil and Switchable Impedance Matching Networks. IEEE Trans. Microw. Theory Tech. 2016, 64, 3714-3722. [CrossRef]

16. Kim, J.; Kim, D.-H.; Choi, J.; Kim, K.-H.; Park, Y.-J. Free-Positioning Wireless Charging System for Small Electronic Devices Using a Bowl-Shaped Transmitting Coil. IEEE Trans. Microw. Theory Tech. 2015, 63, 791-800. [CrossRef]

17. Na, K.; Kim, J.; Park, Y.-J. Free-Positioning Magnetic Resonance Wireless Power Transfer System for Biomedical Devices. In Proceedings of the 2019 IEEE Wireless Power Transfer Conference (WPTC), London, UK, 18-21 June 2019; pp. 497-501. 